\title{
Polarization of a vector boson produced in decay of a heavy fermion in an arbitrary frame
}

\author{
V. Arunprasath \\ Institute of Mathematical Sciences, CIT Campus, Taramani, Chennai, 600 113, India \\ Ritesh K. Singh ${ }^{\dagger}$ \\ Department of Physical Sciences, Indian Institute of Science Education and Research Kolkata, \\ Mohanpur, 741246, India
}

(Received 24 July 2018; published 27 September 2018)

\begin{abstract}
We consider processes where an electroweak gauge boson ( $W$ or $Z$ ) is produced in the decays of a heavy fermion. The polarization state of the produced gauge boson is given in terms of a vector polarization and a rank-2 tensor polarization. In the rest frame of the mother fermion, these are given directly by the dynamical parameters of the underlying theory. In a frame where the mother particle is moving, the polarization parameters of the gauge boson are dependent additionally upon kinematical factors. We show that these kinematical factors depend only on the magnitude of the velocity of the mother particle and derive analytical expressions for them. We apply the results to pair production of heavy fermions at the LHC with one of the fermions decaying to a gauge boson $(W, Z)$ and a light Standard Model fermion. We construct estimators of laboratory frame values of polarization parameters of the produced gauge boson. These estimators can be used to estimate the laboratory frame values of polarization parameters of the produced gauge boson without a detailed simulation of the entire process. We validate our expressions with detailed Monte Carlo simulations in the context of beyond the Standard Model scenarios which have a vectorlike top partner. We also indicate how to include finite width effects of the heavy fermion in some special cases.
\end{abstract}

DOI: 10.1103/PhysRevD.98.053009

\section{INTRODUCTION}

Polarization of unstable particles such as the top, the tau or the electroweak gauge bosons $(W, Z)$ produced in high energy colliders is a powerful tool. It can be used to probe the production or decay of these particles. In particular, the top quark's polarization has been widely suggested as a probe of new physics [1-20]. There exist works on the polarization of tau lepton [21] and the gauge bosons $(W, Z)[22-25]$ as well. The polarization of a particle depends not only on the physics involved in its production but also on the kinematics of its production [26-28]. In this work, we investigate the effect of kinematics on the polarization state of a spin-1 particle, such as $W$ or $Z$, in a class of processes where the boson is produced in the decays of a heavy fermion.

Consider the production of a gauge boson $W$ at the LHC. It can be produced directly: e.g., $p p \rightarrow W^{+} W^{-}$. It can as well be produced in decays of a heavy particle: e.g., $p p \rightarrow t \bar{t}$ with

\footnotetext{
*arunprasath@imsc.res.in

ritesh.singh@iiserkol.ac.in
}

Published by the American Physical Society under the terms of the Creative Commons Attribution 4.0 International license. Further distribution of this work must maintain attribution to the author(s) and the published article's title, journal citation, and DOI. Funded by SCOAP . $t \rightarrow b W$ and $\bar{t}$ decaying inclusively. The kinematics of $W$ production in the second case is different from those in the first case. In the second case, there is a Lorentz boost from the rest frame of the top to the laboratory (lab) frame. This boost arises since the top quark, the parent particle of the $W$, moves with a nonzero velocity in the lab frame. The effect of this boost on the value of $W$ polarization measured in the lab frame is nontrivial. To see this, we first note that the polarization of $W$ is determined by the underlying theory in the rest frame of the top. Since the helicity states of $W$ are not invariant under arbitrary Lorentz boosts (see, e.g., [29,30]), the polarization of $W$ measured in the lab frame is, in general, different from its value in the top quark rest frame.

In this work, we generalize the above mentioned example to include all processes of the type

$$
p p \rightarrow F \bar{F} \rightarrow \bar{F} V f
$$

where $F$ denotes a heavy quark, $V$ a vector boson $(W, Z)$, and $f$ a light quark. ${ }^{1}$ We assume that the $\bar{F}$ decays

\footnotetext{
${ }^{1}$ The restriction to pair production of $F$ is dictated by the requirement that the fermion $F$ be unpolarized (see below). Since the pair production of these vectorlike quarks is dominantly a QCD process, the produced vectorlike quarks are unpolarized. This argument applies to the top quark pair production as well.
} 
inclusively. As will be discussed below, the lab frame value of polarization of the particle is related to that in the rest frame of its mother particle by a kinematical factor. This opens up the possibility of predicting the lab frame polarization, given the theory. In an earlier work [28] we considered the polarization of a spin- $1 / 2$ particle (the top quark) in cases where the particle is produced in the decay of a heavy spin- $1 / 2$ or a spin- 0 particle. This work may be considered as an extension of our previous work to the case of a spin- 1 boson (such as $W$ or $Z$ ) produced in the decay of a heavy quark.

The gauge boson $V$ produced in the decays of the heavy quark $F$ further decays to SM fermions according to its polarization state. Since the vector bosons are spin-1 particles, the polarization state is described by a three component vector $\left[P_{i}(i=x, y, z)\right]$ and a five component tensor polarization $\left(T_{x y}, T_{y z}, T_{z z}, T_{x x}-T_{y y}, T_{x z}\right)$. In the rest frame of the heavy quark $F$, the polarization parameters of $V$ are given by dynamical parameters such as couplings, mixing matrices and masses of the particles involved in the decay. On the other hand, the lab frame values of polarization parameters of $V$ are simpler to be measured in experiments as they do not require the full reconstruction of the entire event.

To connect the experimentally measured values of polarization parameters with theory parameters (the dynamical parameters relevant in the decay of $F$ ), one requires a method to predict the lab frame values of the polarization parameters from a theory. To achieve this, we propose estimators for lab frame polarization parameters of $V$ [28].

These estimators (for the eight polarization parameters of $V$ ) are of the following form:

$$
\mathcal{P}_{\text {estimator }} \equiv \frac{1}{\sigma_{F F}} \int \frac{d \sigma_{F F}}{d \beta_{F}} \mathcal{P}\left(\beta_{F}\right)
$$

Here, $\sigma_{F F}$ is the cross section for the pair production of the parent quark $F: p p \rightarrow F \bar{F}$, and $\beta_{F}$ is the velocity of $F$ in the lab frame. $\mathcal{P}\left(\beta_{F}\right)$ can be interpreted as the polarization parameter of $V$ in a single event. Then the above expression can be interpreted as a weighted average of a polarization parameter of $V$ over the entire sample of events. The weighting factor $\frac{d \sigma_{F F}}{d \beta_{F}}$ includes convolution over the parton distribution functions. Note that these estimators require only the magnitude of velocity of its mother particle. Hence, these estimators are simple to use in a Monte Carlo simulation of the relevant process. An advantage of the use of these estimators is that one can now predict the value of polarization parameters of $V$ without any simulation of the decay of $F$, its mother particle. These estimators are derived under the assumption that the parent quark has a narrow width $\left(\Gamma / m_{F} \ll 1\right)$. We shall later relax this assumption and consider the case of larger widths of the mother particle in Sec. V. We find that these estimators reproduce the polarization parameters of $V$ to within a few percent in the narrow width case. In the finite width case, when the nonresonant and off-shell contributions are small, we modify these estimators to include the Breit-Wigner shape of the propagator of $F$. We find that both the original and the modified estimators provide equally good approximations to the polarization parameters of $V$, in the finite width case.

The processes mentioned above are possible in models with heavy fermions with electroweak gauge couplings. The Standard Model top quark pair production and decay $(p p \rightarrow t \bar{t} \rightarrow \bar{t} b W)$ also belongs to this class of processes. The heavy fermion $F$ may belong to a chiral or a vectorlike representation of the electroweak gauge group. In this work, we take either $F$ to be a hypothetical vectorlike quark or the top quark. ${ }^{2}$ Examples of such processes involving vectorlike fermions are: $p p \rightarrow T \bar{T} \rightarrow \bar{T} Z t$ and $p p \rightarrow$ $B \bar{B} \rightarrow \bar{B} t W$ where $T(B)$ is a vectorlike quark with charge $+2 / 3(-1 / 3)$. The formalism used in this work is applicable to any model of heavy fermions with such decay processes.

Vectorlike fermions appear naturally in various beyond the SM scenarios which address the hierarchy problem in the Higgs sector: warped extra-dimension models [36], composite Higgs models [37-39] and Little Higgs models [40-45], for example. There is a renewed interest in the phenomenology of vectorlike fermions. Recently, a number of studies that were proposed to explain possible evidence of a $750 \mathrm{GeV}$ resonance decaying to a diphoton final state $[46,47]$ considered models with additional vectorlike fermions (see, e.g., [48-52]).

The vectorlike fermions can have a bare mass term unlike the chiral fermions of the SM. As a result, one can have heavy vectorlike fermions without a large coupling to Higgs (if allowed by symmetry). This leads to the decoupling of their effects in electroweak oblique corrections, Higgs production and decay, in the limit where their mass goes to infinity, with their coupling to Higgs (if any) remains fixed. Perturbative unitarity bounds can be also evaded for any given large mass of a vectorlike fermion, for an appropriately small mixing with the SM fermions [53].

Though vectorlike fermions can appear in various representations of the SM gauge group, the strongly interacting fermions of charge $+2 / 3$ and $-1 / 3$, the socalled top quark and bottom quark partners, play important roles in the models mentioned above. For example, in Little Higgs models, the quadratic divergence in Higgs selfenergy coming from the top quark loop is canceled by the contribution of top partners.

\footnotetext{
${ }^{2}$ The simplest model involving additional chiral heavy fermions, the fourth generation SM, has been ruled out by the discovery of the Higgs at the LHC [31]. The reason for the exclusion is the accidental suppression of $h \rightarrow \gamma \gamma$ rate by two orders of magnitude relative to the SM value, in this model. However, the possibility of the existence of fermions in vectorlike representations of the SM gauge group is not excluded, due to the decoupling properties of vectorlike fermions [32-35].
} 
The masses of these vectorlike quarks are constrained by the direct searches at the LHC $[54,55]$. The assumption is that they couple only to third-generation SM quarks. In general, vectorlike quarks can couple to the first two generations of SM fermions as well [33]. Depending upon the assumptions on the branching ratios, the lowest direct search bounds from the LHC read $790 \mathrm{GeV}$ for the charge $+2 / 3$ quark, $730 \mathrm{GeV}$ for the charge $-1 / 3$ quark [55].

Flavor observables, precision electroweak observables, Higgs coupling measurements constrain indirectly the mixing of the top quark (or bottom quark) partners with the SM quarks and their masses. The constraints depend upon the representation of these vectorlike quarks under the SM gauge group [32-35].

This paper is divided into six sections with the first section being the introduction to this work. Section II describes the expressions of polarization parameters of $V$ in the rest frame of $F$. Section III discusses two models of a vectorlike quark $T$ of charge $+2 / 3$. In this section, benchmark values of model parameters are provided and the corresponding $Z$ polarization parameters are obtained for the decay $T \rightarrow Z t$.
Section IV describes the formalism for the derivation of the polarization estimators. Section V completes the derivation of polarization estimators of $V$ at the level of $p p$ collisions and describes numerical validation of the polarization estimators. Section VI presents a summary of this work.

\section{POLARIZATION PARAMETERS OF $V$}

In this section, we give expressions for the polarization parameters of the vector boson $V$, in the rest frame of the parent particle $F$ in the decay $F \rightarrow V f$. The only nonvanishing polarization parameters are $P_{z}$ and $T_{z z}$ and they are defined by

$$
P_{z}=\frac{\Gamma^{+}-\Gamma^{-}}{\Gamma^{+}+\Gamma^{-}+\Gamma^{0}}, \quad T_{z z}=\frac{\Gamma^{+}-2 \Gamma^{0}+\Gamma^{-}}{\Gamma^{+}+\Gamma^{-}+\Gamma^{0}},
$$

where $\Gamma^{i}(i=+, 0,-)$ denote the partial decay widths corresponding to the decay of $F$ into an unpolarized $f$ and an electroweak gauge boson $V$ with helicity $i$. Taking the vertex $F f V$ vertex as $\gamma^{\mu}\left(g_{L} P_{L}+g_{R} P_{R}\right)$ where $P_{L}$ and $P_{R}$ are the left and right chiral projectors, we get

$$
\begin{aligned}
P_{z} & =\frac{2\left(g_{R}^{2}-g_{L}^{2}\right) K^{1 / 2} \xi_{V}}{-12 \xi_{V} \sqrt{\xi_{f}} g_{L} g_{R}+\left(g_{L}^{2}+g_{R}^{2}\right)\left(K+3 \xi_{V}\left(1+\xi_{f}-\xi_{V}\right)\right)}, \\
T_{z z} & =-\sqrt{\frac{2}{3}} \frac{\left(g_{R}^{2}+g_{L}^{2}\right) K}{\left(-12 \xi_{V} \sqrt{\xi_{f}} g_{L} g_{R}+\left(g_{L}^{2}+g_{R}^{2}\right)\left(K+3 \xi_{V}\left(1+\xi_{f}-\xi_{V}\right)\right)\right)}
\end{aligned}
$$

where $\xi_{V}=m_{V}^{2} / m_{F}^{2}, \xi_{f}=m_{f}^{2} / m_{F}^{2}$ and $K \equiv K\left(1, \xi_{V}, \xi_{f}\right)$ with $K(x, y, z)=x^{2}+y^{2}+z^{2}-2 x y-2 y z-2 z x . m_{F}, m_{V}$ and $m_{f}$ denote the masses of the particles $F, V$ and $f$, respectively. Note that $T_{z z}$ is nonzero even when $g_{L}=g_{R}$.

\section{MODELS}

In this section we consider the models which have a vectorlike quark $(T)$ of charge $+2 / 3$ mixing with the thirdgeneration quarks of the SM [32,34]. The models depend upon the representation of $T$ under the SM gauge group. We consider the cases where $T$ is an electroweak singlet, and where $T$ forms an electroweak doublet with a vectorlike quark $B$ of charge $-1 / 13$. We refer to them as the singlet model and the doublet model, respectively. In both the models, we consider the decay: $T \rightarrow Z t$ where $t$ is the top quark.

\section{A. The singlet model}

The Yukawa part of the Lagrangian reads

$$
\mathcal{L}=-y_{t} \bar{q}_{L}^{\prime} \tilde{\Phi} t_{R}^{\prime}-\lambda \bar{q}_{L}^{\prime} \tilde{\Phi} T_{R}^{\prime}-M \bar{T}_{L}^{\prime} T_{R}^{\prime}+\text { H.c. }
$$

The Yukawa coupling of the top is denoted as $y_{t}$. We have not included the Yukawa part of the SM in the above equation. The primes refer to the fact that the Lagrangian is in gauge basis. $\Phi$ is the Higgs doublet with $\tilde{\Phi}=i \tau_{2} \Phi^{*}\left(\tau_{2}\right.$ is a Pauli matrix, $*$ denotes complex conjugation). After the Higgs doublet acquires a vacuum expectation value (vev), $\langle\Phi\rangle=(0, v / \sqrt{2})^{\prime}$ (the prime denotes transpose), $T$ mixes with the SM fermions with the amount of mixing determined by $\lambda$. Neglecting the top mixing with the first two generation fermions, we get two mass eigenstates $(t, T)$ by performing a bidiagonalization of the mass matrix. Taking the mass eigenvalues as the physical masses of the top and $T, m_{t}$ and $m_{T}$, respectively, we have

$$
\begin{aligned}
\frac{y_{t}^{2} v^{2}}{2} & =m_{t}^{2}\left(1+\frac{x^{2}}{M^{2}-m_{t}^{2}}\right), \quad m_{T}^{2}=M^{2}\left(1+\frac{x^{2}}{M^{2}-m_{t}^{2}}\right), \\
\sin \theta_{L} & =\frac{M x}{\sqrt{\left(M^{2}-m_{t}^{2}\right)^{2}+M^{2} x^{2}}}, \quad \sin \theta_{R}=\frac{m_{t}}{M} \sin \theta_{L},
\end{aligned}
$$

where $x=\lambda v / \sqrt{2} . \theta_{L}$ and $\theta_{R}$ are the mixing angles of the left and right chiral parts of the top and $T$, respectively, obtained after the bidiagonalization of the top- $T$ mass matrix. We take $m_{t}, m_{T}$ and $\theta_{L}$ as the independent parameters. Constraints on this model coming from 
contribution to oblique parameters $S, T$ and $U$, loop level corrections to $Z b \bar{b}$ vertex, in the form of upper bound on the mixing read $\sin \theta_{L}<0.15$, for $m_{T}>750 \mathrm{GeV}$ at 95\% C.L. [34].

\section{B. The doublet model}

The Yukawa part of the Lagrangian reads

$$
\begin{aligned}
\mathcal{L}= & -y_{t} \bar{q}_{L}^{\prime} \tilde{\Phi} t_{R}^{\prime}-\lambda_{t} \bar{q}_{L}^{\prime} \tilde{\Phi} t_{R}^{\prime}-\lambda_{d} \bar{Q}_{L}^{\prime} \Phi b_{R}^{\prime} \\
& -y_{b} \bar{q}_{L}^{\prime} \Phi b_{R}^{\prime}-M \bar{Q}_{L} Q_{R}^{\prime}+\text { H.c. },
\end{aligned}
$$

where $Q=(T, B)$ is a doublet of vectorlike quarks and $y_{b}$ denotes the Yukawa coupling of the bottom quark. $\lambda_{t}$ and $\lambda_{b}$ determine the $t-T$ and $b-B$ mixing, respectively. We have neglected the mixing of third-generation quarks with the corresponding quarks of the first two generations as we are not interested in the effects on flavor observables. After the Higgs gets a vev, the top and $T$ mix, and $b$ and $B$ mix as well. With $x=\lambda_{t} v / \sqrt{2}$ and $x_{b}=\lambda_{b} v / \sqrt{2}$, we have

$$
\begin{aligned}
\sin \theta_{R}^{u} & =\frac{M x_{t}}{\sqrt{\left(M^{2}-m_{t}^{2}\right)^{2}+M^{2} x_{t}^{2}}}, \quad \sin \theta_{L}^{u}=\frac{m_{t}}{M} \sin \theta_{R}^{u}, \\
\frac{y_{t}^{2} v^{2}}{2} & =m_{t}^{2}\left(1+\frac{x_{t}^{2}}{M^{2}-m_{t}^{2}}\right), \quad m_{T}^{2}=M^{2}\left(1+\frac{x_{t}^{2}}{M^{2}-m_{t}^{2}}\right), \\
\sin \theta_{R}^{d} & =\frac{M x_{b}}{\sqrt{\left(M^{2}-m_{b}^{2}\right)^{2}+M^{2} x_{b}^{2}}}, \quad \sin \theta_{L}^{d}=\frac{m_{b}}{M} \sin \theta_{R}^{d} \\
\frac{y_{b}^{2} v^{2}}{2} & =m_{b}^{2}\left(1+\frac{x_{b}^{2}}{M^{2}-m_{b}^{2}}\right), \quad m_{B}^{2}=M^{2}\left(1+\frac{x_{b}^{2}}{M^{2}-m_{b}^{2}}\right),
\end{aligned}
$$

where $\theta_{L, R}^{u}$ and $\theta_{L, R}^{d}$ denote mixing angles of the left chiral and right chiral parts of the $t-T$ and $b-B$ pairs, respectively, and $m_{b}$ and $m_{B}$ denote the masses of the bottom quark and the $B$ quark, respectively. Constraints from the appearance of tree-level corrections to the $Z b \bar{b}$ vertex, contribution to oblique parameters and loop level contributions to $Z b \bar{b}$ vertex read $\sin \theta_{R}^{d}<0.06$ and $\sin \theta_{R}^{u}<0.13$ for $m_{T}>$ $750 \mathrm{GeV}$ at $95 \%$ C.L. [34]. The mass splitting between $B$ and $T$ is also severely constrained to lie within a few $\mathrm{GeVs}$ [34]. We assume that $B$ is degenerate in mass with $T$ : $m_{B}=m_{T}$. In this model, there are three free parameters: $m_{T}, \theta_{R}^{d}$ and $\theta_{R}^{u}$.

\section{Benchmark points}

In this subsection, we describe our choices of parameter values that are used throughout this work. In the case of the singlet model and the doublet model, we take the lower bound of the mass of $T$ as $900 \mathrm{GeV}$, based on the current direct search constraints. We take $\theta_{L}=0.1$ in the singlet model due to the constraints mentioned in the previous section. We set $m_{B}=m_{T}, \theta_{R}^{u}=0.09$ and $\theta_{R}^{d}=0.05$ in the
TABLE I. List of parameters and their values in the two models, the singlet model and the doublet model.

\begin{tabular}{lcc}
\hline \hline Parameter & Singlet & Doublet \\
\hline$m_{T}(\mathrm{GeV})$ & $\geq 900$ & $\geq 900$ \\
$m_{B}(\mathrm{GeV})$ & $=m_{T}$ & $=m_{T}$ \\
Mixing angle(s) & $\theta_{L}=0.1$ & $\theta_{R}^{u}=0.09$ \\
& & $\theta_{R}^{d}=0.05$ \\
\hline \hline
\end{tabular}

doublet Model, to be consistent with the constraints. The values are tabulated in Table I.

\section{Discussion}

We now discuss the value of $P_{z}$ and $T_{z z}$ for specific models described above for the decay $T \rightarrow Z t$, and $t \rightarrow b W$ in the $\mathrm{SM}$. In the case of the singlet model, the coupling $Z t T$, which is responsible for the decay $T \rightarrow Z t$, is purely left chiral: $g_{L}=\left(g /\left(2 \cos \theta_{W}\right)\right) \sin \theta_{L} \cos \theta_{L}$ and $g_{R}=0$ where $\theta_{W}$ denotes the weak mixing angle and $g$ the $S U(2)_{L}$ gauge coupling. In the case of the doublet model, the $Z t T$ vertex is purely right chiral: $g_{L}=0, g_{R}=-\left(g /\left(2 \cos \theta_{W}\right)\right) \times$ $\cos \theta_{R}^{u} \sin \theta_{R}^{u}$. For these two models, the expressions for $P_{z}$ and $T_{z z}$ can be obtained by the following replacements: $\xi_{V} \rightarrow \xi_{Z}=m_{Z}^{2} / m_{T}^{2}, \xi_{f} \rightarrow \xi_{t}=m_{t}^{2} / m_{T}^{2}$ and substituting the values of $g_{L}$ and $g_{R}$ in Eq. (4).

In the case of the SM top decay, the coupling is purely left chiral: $g_{L}=g / \sqrt{2}$ and $g_{R}=0$. The expression for $P_{z}$ and $T_{z z}$ of $W$ can be obtained by the following replacements $\xi_{V} \rightarrow \xi_{W}=m_{W}^{2} / m_{t}^{2}, \xi_{f} \rightarrow \xi_{b}=m_{b}^{2} / m_{t}^{2}$ and substituting the values of $g_{L}$ and $g_{R}$ in Eq. (4).

\section{FORMALISM}

To obtain the expressions for the polarization estimators, we begin by looking at the parton-level process of the form

$$
p_{1} p_{2} \rightarrow F \bar{F} \rightarrow \bar{F} V f,
$$

where $p_{1}, p_{2}$ denote partons. We shall assume that the produced $V$ further decays to leptons: $V \rightarrow \ell \bar{\ell}^{\prime}$. The parton-level amplitude can be written as

$$
\begin{aligned}
i \mathcal{M}= & \frac{1}{\left(p_{F}^{2}-m_{F}^{2}\right)+i m_{F} \Gamma_{F}} \frac{1}{\left(p_{V}^{2}-m_{V}^{2}\right)+i m_{V} \Gamma_{V}} \\
& \times i \mathcal{M}^{\prime}\left(V \rightarrow \ell \bar{\ell}^{\prime}\right)_{\mu}\left(-i P^{\mu \nu}\right) \\
& \times i \mathcal{M}^{\prime}(F \rightarrow f V)_{\nu \alpha}\left(i P_{\alpha \beta}\right) \\
& \times i \mathcal{M}^{\prime}(q \bar{q} / g g \rightarrow F \bar{F})_{\beta},
\end{aligned}
$$

where the primes on amplitudes denote that the wave functions of the particles which are exchanged have not been included. The quantities $P^{\mu \nu}$ and $P_{\alpha \beta}$ denote the numerators of the propagators of $V$ and $F$, respectively. The indices $\alpha$ and $\beta(\alpha, \beta=1, \cdots 4)$ denote the components of 
the spinors and Dirac matrices. For clarity, the denominators of the propagators have been explicitly taken out of the expressions for the amplitudes. We have

$$
\begin{array}{rlrl}
P^{\mu \nu} & =-\sum_{i} \hat{\epsilon}\left(p_{V}^{\mathrm{PCM}}\right)^{(i)} \hat{\epsilon}\left(p_{V}^{\mathrm{PCM}}\right)^{(i) *}, & & i=-1,0,1, \\
P_{\alpha \beta} & =\sum_{\lambda} u\left(p_{F}^{\mathrm{PCM}}, \lambda\right)_{\alpha} \bar{u}\left(p_{F}^{\mathrm{PCM}}, \lambda\right)_{\beta}, & \lambda=-1 / 2,+1 / 2,
\end{array}
$$

where $i, \lambda$ and $p_{F}^{\mathrm{PCM}}, p_{V}^{\mathrm{PCM}}$ are the helicities and momenta of the particles $F$ and $V$, respectively, measured in the parton center of mass (PCM) frame. Substituting these relations in Eq. (10), and defining

$$
\begin{aligned}
& i \mathcal{M}_{F \bar{F}}\left(p_{F}^{\mathrm{PCM}}, p_{\bar{F}}^{\mathrm{PCM}}\right)_{\lambda} \\
& \quad=\sum_{\beta} \bar{u}\left(p_{F}^{\mathrm{PCM}}, \lambda\right)_{\beta} i \mathcal{M}^{\prime}(q \bar{q} / g g \rightarrow F \bar{F})_{\beta}, \\
& i \mathcal{M}_{f F V}\left(p_{F}^{\mathrm{PCM}}, p_{f}^{\mathrm{PCM}}, p_{V}^{\mathrm{PCM}}\right)_{i \lambda} \\
& \quad=\sum_{\alpha} i \mathcal{M}^{\prime}(F \rightarrow f V)_{\nu \alpha} \hat{\epsilon}^{\nu(i) *}\left(p_{V}^{\mathrm{PCM}}\right) u\left(p_{F}^{\mathrm{PCM}}, \lambda\right)_{\alpha}, \\
& i \mathcal{M}_{V \ell \bar{\ell}^{\prime}}\left(p_{V}^{\mathrm{PCM}}, p_{\ell}^{\mathrm{PCM}}, p_{\bar{\ell}^{\prime}}^{\mathrm{PCM}}\right)^{(i)} \\
& \quad=\sum_{\mu} i \mathcal{M}^{\prime}\left(V \rightarrow \ell \bar{\ell}^{\prime}\right)_{\mu} \hat{\epsilon}^{\mu(i)}\left(p_{V}^{\mathrm{PCM}}\right),
\end{aligned}
$$

we write the amplitude as

$$
\begin{aligned}
i \mathcal{M} \sim & i \mathcal{M}_{F \bar{F}}\left(p_{F}^{\mathrm{PCM}}, p_{\bar{F}}^{\mathrm{PCM}}\right)_{\lambda} i \mathcal{M}_{f F V}\left(p_{F}^{\mathrm{PCM}}, p_{f}^{\mathrm{PCM}}, p_{V}^{\mathrm{PCM}}\right)_{\lambda}^{(i)} \\
& \times i \mathcal{M}_{V \ell \bar{\ell}^{\prime}}\left(p_{V}^{\mathrm{PCM}}, p_{\ell}^{\mathrm{PCM}}, p_{\bar{\ell}^{\prime}}^{\mathrm{PCM}}\right)^{(i)} .
\end{aligned}
$$

The phase space element of the parton-level process can be written in terms of 2-body phase space elements as

$$
d \Phi=\frac{d p_{V}^{2}}{2 \pi} \frac{d p_{F}^{2}}{2 \pi} d \Phi_{F \bar{F}} d \Phi_{f F V} d \Phi_{V \ell \bar{\ell}^{\prime}}
$$

with

$$
\begin{aligned}
d \Phi_{F \bar{F}} & =(2 \pi)^{4} \delta^{(4)}\left(P_{\mathrm{in}}-p_{F}-p_{\bar{F}}\right) \frac{d^{3} p_{F}}{(2 \pi)^{3} 2 E_{F}} \frac{d^{3} p_{\bar{F}}}{(2 \pi)^{3} 2 E_{\bar{F}}}, \\
d \Phi_{f F V} & =(2 \pi)^{4} \delta^{(4)}\left(P_{F}-p_{f}-p_{V}\right) \frac{d^{3} p_{f}}{(2 \pi)^{3} 2 E_{f}} \frac{d^{3} p_{V}}{(2 \pi)^{3} 2 E_{V}}, \\
d \Phi_{V \ell \bar{\ell}^{\prime}} & =(2 \pi)^{4} \delta^{(4)}\left(P_{V}-p_{\ell}-p_{\bar{\ell}^{\prime}}\right) \frac{d^{3} p_{\ell}}{(2 \pi)^{3} 2 E_{\ell}} \frac{d^{3} p_{\bar{\ell}^{\prime}}}{(2 \pi)^{3} 2 E_{\bar{\ell}^{\prime}}},
\end{aligned}
$$

where $P_{\text {in }}$ denotes the sum of initial state parton momenta and $p_{F}^{2}, p_{V}^{2}$ denote the invariant masses of $F$ and $V$, respectively, $\left(p_{F}^{2}>0, p_{V}^{2}>0\right)$. Squaring the amplitude $i \mathcal{M}$, multiplying with the phase space element and flux factor, and averaging over initial state spin and color indices, summing over intermediate state spin and color indices, we get the parton-level cross section for the process as

$$
\hat{\sigma}=\frac{f_{\mathrm{avg}}^{\mathrm{in}}}{2 E_{1} 2 E_{2}\left|\vec{v}_{1}-\vec{v}_{2}\right|} \int \Delta_{\mathrm{BW}}\left(p_{V}^{2}\right) \Delta_{\mathrm{BW}}\left(p_{F}^{2}\right) \sum|\mathcal{M}|^{2} d \Phi,
$$

where $f_{\mathrm{avg}}^{\text {in }}$ denotes the spin and color averaging factor for the initial state and $E_{i}, \vec{v}_{i}(i=1,2)$ denote the energies and velocities of the initial state partons. In the above equation, $\Delta_{\mathrm{BW}}\left(p^{2}\right)=\left(\left(p^{2}-m^{2}\right)+m^{2} \Gamma^{2}\right)^{-1}$ denote the Breit-Wigner factors arising from the propagators. When the width of an intermediate particle is much smaller than its width, $\Delta_{\mathrm{BW}}\left(p^{2}\right)$ can be replaced by a Dirac delta function as follows ${ }^{3}$ :

$$
\Delta_{\mathrm{BW}}\left(p^{2}\right) \rightarrow \frac{\pi}{m \Gamma} \delta\left(p^{2}-m^{2}\right) .
$$

Assuming that this approximation, the so-called narrowwidth approximation (NWA), holds for both $F$ and $V$, using the factorized form of the phase space element, we write Eq. (16) as

$$
\hat{\sigma}=f_{\text {avg }}^{V} f_{\text {avg }}^{F} \int \sum_{\{\lambda, i\}} \hat{\sigma}_{\lambda^{\prime} \lambda}^{F \bar{F}} \frac{d \Gamma_{\lambda \lambda^{\prime}, i^{\prime} i}^{F f}}{\Gamma_{F}^{V}} \frac{d \Gamma_{i i^{\prime}}^{V \ell \bar{t}^{\prime}}}{\Gamma_{V}},
$$

where $\int \sum_{\lambda} d \sigma_{\lambda \lambda}^{F \bar{F}}=\hat{\sigma}^{F \bar{F}}$, the cross section for the pair production of $F, \int \sum_{(\lambda, i)} d \Gamma_{\lambda \lambda ; i i}^{F f V}$ is the partial decay width for the decay $F \rightarrow f V, \int \sum_{i} d \Gamma_{i i}^{V \ell} \bar{\ell}^{\prime}$ is the partial decay width for the decay $V \rightarrow \ell \bar{\ell}^{\prime}$ and $f_{\mathrm{avg}}^{V}$ and $f_{\mathrm{avg}}^{F}$ denote the spin averaging factors included in the definitions of $d \Gamma^{V}$ and $d \Gamma^{F}$.

The Lorentz invariance of the phase space element factors allows evaluation of different parts of the squared amplitude in different frames. The pair production of $F$ can be evaluated in the PCM frame, the decay of $F$ in the rest frame of $F$ and the decay of $V$ in the rest frame of $V$. The rest frame of $V$ can be reached from the PCM frame through the transformation

$$
h^{-1}\left(p_{V}^{\mathrm{PCM}}\right) \equiv \Lambda_{z}^{-1}\left(\beta_{V}^{\mathrm{PCM}}\right) R^{-1}\left(\theta_{V}^{\mathrm{PCM}}, \phi_{V}^{\mathrm{PCM}}\right)
$$

where $\beta_{V}^{\mathrm{PCM}}, \theta_{V}^{\mathrm{PCM}}$ and $\phi_{V}^{\mathrm{PCM}}$ define the velocity and the direction of motion of $V$ in the PCM frame,

PCM frame $\stackrel{h^{-1}\left(p_{V}^{\mathrm{PCM}}\right)}{\longrightarrow} V$ rest frame.

The rest frame of $F$ can be reached from the PCM frame by a Lorentz transformation

$$
h^{-1}\left(p_{F}^{\mathrm{PCM}}\right) \equiv \Lambda_{z}^{-1}(\bar{\beta}) R^{-1}(\bar{\theta}, \bar{\phi}),
$$

where $\bar{\beta}, \bar{\theta}, \bar{\phi}$ define the velocity and the direction of motion of $F$ in the PCM frame,

\footnotetext{
${ }^{3}$ This approximation breaks down when the mass difference between the mother particle and any one of the daughter particles is of the order of the width of the mother particle [56-58].
} 
PCM frame $\stackrel{h^{-1}\left(p_{F}^{\mathrm{PCM}}\right)}{\longrightarrow} F$ rest frame.

This transformation transforms the momenta to the rest frame of $F: p_{V}^{\mathrm{PCM}} \rightarrow p_{V}^{F}, p_{f}^{\mathrm{PCM}} \rightarrow p_{f}^{F}$. Under this transformation, the helicities of $F$ are unchanged as the Lorentz transformation is along its direction of motion. On the other hand, the helicity states of $V$ transform in the following way:

$\hat{\epsilon}^{(i)}\left(p_{V}^{\mathrm{PCM}}\right) \stackrel{h^{-1}\left(p_{F}^{\mathrm{PCM}}\right)}{\longrightarrow} R_{k i}(\mathcal{R}) \hat{\epsilon}^{(k)}\left(p_{V}^{F}\right) \quad(i, k=+, 0,-)$,

where $R$ is a rotation matrix corresponding to the rotation

$$
\mathcal{R}=h^{-1}\left(p_{V}^{F}\right) h^{-1}\left(p_{F}^{\mathrm{PCM}}\right) h\left(p_{V}^{\mathrm{PCM}}\right) .
$$

In the above expressions, $p_{V}^{F}=\Lambda_{z}^{-1}(\bar{\beta}) R^{-1}(\bar{\theta}, \bar{\phi}) p_{V}^{\mathrm{PCM}}$ is the momentum of $V$ in the rest frame of $F$, and $p_{V}^{\mathrm{PCM}}$ and $p_{F}^{\mathrm{PCM}}$ denote the PCM frame momenta of $F$ and $V$, respectively. ${ }^{4}$ The expression for the matrix element after the transformations have been applied becomes

$$
\begin{aligned}
i \mathcal{M}= & \sum_{\lambda, k, i} i \mathcal{M}_{F \bar{F}}\left(p_{F}^{\mathrm{PCM}}, p_{\bar{F}}^{\mathrm{PCM}}\right)_{\lambda} R_{k i} i \mathcal{M}_{f F V}\left(p_{F}^{F}, p_{f}^{F}, p_{V}^{F}\right)_{\lambda}^{(k)} i \\
& \times \mathcal{M}_{V \ell \bar{\ell}^{\prime}}\left(p_{V}^{V}, p_{\ell}^{V}, p_{\bar{\ell}^{\prime}}^{V}\right)^{(i)}
\end{aligned}
$$

This implies that the expression for the parton-level cross section for the process becomes

$\hat{\sigma}=f_{\mathrm{avg}}^{F} f_{\mathrm{avg}}^{V} \times \int \sum_{\{\lambda\},\{i\}} d \sigma_{\lambda^{\prime} \lambda}^{F \bar{F}} R_{i^{\prime} k^{\prime}}^{\dagger}\left(\frac{d \Gamma_{\lambda \lambda^{\prime} k^{\prime} k}^{F f V}}{\Gamma_{F}}\right)_{F} R_{k i}\left(\frac{d \Gamma_{i i^{\prime}}^{V e \overline{\prime^{\prime}}}}{\Gamma_{V}}\right)_{V}$,

where $\{\lambda\}$ and $\{i\}$ denote the set of helicity indices corresponding to $F$ and $V$ that appear in the above expression. In the above expression, the subscripts $F$ and $V$ on $d \Gamma^{f F V} / \Gamma_{F}$ and $d \Gamma^{V \ell} \bar{\ell}^{\prime} / \Gamma_{V}$ indicate that they have to be evaluated in their respective frames. We assume that the production of the heavy fermion pair is through QCD interactions. Since QCD conserves parity, the produced fermion $F$ is unpolarized. This allows the following simplification, after a partial integration over the $F \bar{F}$ phase space:

$$
d \sigma_{\lambda^{\prime} \lambda} \rightarrow \frac{1}{f_{\mathrm{avg}}^{F}} \frac{d \sigma_{F \bar{F}}}{d \bar{\Omega}} \delta_{\lambda^{\prime} \lambda} d \bar{\Omega}
$$

\footnotetext{
${ }^{4}$ To see that the transformation in Eq. (18) defines a rotation, consider the application of the transformations on the momentum

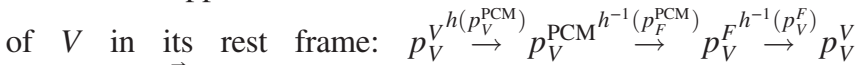
$\left[p_{V}^{V}=\left(m_{V}, \overrightarrow{0}\right)\right]$. The transformation takes the rest frame of $V$ to itself. Hence, it is a rotation on the rest frame of $V$. This can also be checked explicitly using the expressions for the Lorentz transformations.
}

where $f_{\text {avg }}^{F}=2$ and $d \sigma_{F \bar{F}} / d \bar{\Omega}$ is the differential cross section for the production of the $F \bar{F}$ pair, in the PCM frame. Substituting this in Eq. (26), we get

$\hat{\sigma}=f_{\mathrm{avg}}^{V} \times \int \frac{d \sigma_{F \bar{F}}}{d \bar{\Omega}} \sum_{\{i\}} R_{i^{\prime} k^{\prime}}^{\dagger}\left(\frac{d \Gamma_{\lambda \lambda^{\prime} ; k^{\prime} k}^{F f V}}{\Gamma_{F}}\right)_{F} R_{k i}\left(\frac{d \Gamma_{i i^{\prime}}^{V \ell \bar{\ell}^{\prime}}}{\Gamma_{V}}\right)_{V} d \bar{\Omega}$.

In the rest frame of $V$, the matrix $d \Gamma^{V \ell \bar{\ell}^{\prime}}$ is given, after partial integration over phase space, by

$$
\frac{d \Gamma^{V \ell \bar{\ell}^{\prime}}}{d \Omega_{\ell}^{V}}=\Gamma^{V \ell \bar{\ell}^{\prime}} \frac{\rho_{i i^{\prime}}^{V}}{4 \pi},
$$

where $d \Omega_{\ell}^{V}=\sin \theta_{\ell} d \theta_{\ell} d \phi_{\ell}$ and $\theta, \phi$ define the direction of motion of $\ell$ in the rest frame of $V$. The matrix $\rho^{V}$ is a matrix with $\sum_{i} \rho_{i i}^{V}=1$ and can be parametrized as follows:

$$
\rho^{V}=\left(\begin{array}{ccc}
\frac{1+c_{\theta_{\ell}}^{2}+2 \alpha c_{\theta_{\ell}}}{4} & \frac{s_{\theta_{\ell}}\left(\alpha+c_{\theta_{\ell}}\right)}{2 \sqrt{2}} e^{i \phi_{\ell}} & \frac{\left(1-c_{\theta_{\ell}}^{2}\right)}{4} e^{2 i \phi_{\ell}} \\
\frac{s_{\theta_{\ell}}\left(\alpha+c_{\theta_{\ell}}\right)}{2 \sqrt{2}} e^{-i \phi_{\ell}} & \frac{s_{\theta_{\ell}}^{2}}{2} & \frac{s_{\theta_{\ell}}\left(\alpha-c_{\theta_{\ell}}\right)}{2 \sqrt{2}} e^{i \phi_{\ell}} \\
\frac{\left(1-c_{\theta_{\ell}}^{2}\right)}{4} e^{-2 i \phi_{\ell}} & \frac{s_{\theta_{\ell}}\left(\alpha-c_{\theta_{\ell}}\right)}{2 \sqrt{2}} e^{-i \phi_{\ell}} & \frac{1+c_{\theta_{\ell}}^{2}-2 \alpha c_{\theta_{\ell}}}{4}
\end{array}\right)
$$

where $s_{x}=\sin x, c_{x}=\cos x$. In the above equation, $\alpha=$ $\left(\left(g_{L}^{\ell}\right)^{2}-\left(g_{R}^{\ell}\right)^{2}\right) /\left(\left(g_{L}^{\ell}\right)^{2}+\left(g_{R}^{\ell}\right)^{2}\right)$ with $g_{L}^{\ell}$, and $g_{R}^{\ell}$ denoting the left and right chiral couplings of the $V \ell \bar{\ell}^{\prime}$ vertex.

In the case where the polarization of $V$ is measured in the rest frame of $F$ obtained by a sequence of Lorentz transformations starting from the PCM frame,

PCM frame $\stackrel{h^{-1}\left(p_{F}^{\mathrm{PCM}}\right)}{\longrightarrow} \mathrm{F}$ rest frame $\stackrel{h^{-1}\left(p_{F}^{F}\right)}{\longrightarrow} \mathrm{V}$ rest frame,

the helicity states of $V$ do not undergo helicity rotation. In this case,

$$
\left(\frac{d \Gamma^{F f V}}{d \Omega^{F}}\right)_{F}=\Gamma^{F f V} \frac{\rho_{i^{\prime} i}^{F}}{4 \pi},
$$

where $\Gamma^{F f V}$ is the partial width for the decay $F \rightarrow f V, \rho^{F}$ is a matrix with constant elements, and $\sum_{i} \rho_{i i}^{F}=1$. In this case, the expression for $\hat{\sigma}$ becomes

$$
\begin{aligned}
\hat{\sigma}= & \int \sigma_{F \bar{F}} \times \frac{f_{\mathrm{avg}}^{V}}{4 \pi} \sum_{\{i\}} \rho_{i^{\prime} i}^{F} \rho_{i i^{\prime}}^{V} d \Omega_{\ell}^{V} \times B . R(F \rightarrow V f) \\
& \times B . R\left(V \rightarrow \ell \bar{\ell}^{\prime}\right),
\end{aligned}
$$

where B.R denotes a branching ratio. From the above expression, differential angular distribution of $\ell$ in the rest frame of $V$ can be obtained,

$$
\frac{1}{\sigma} \frac{d \sigma}{d \Omega_{\ell}^{V}}=\frac{f_{\mathrm{avg}}^{V}}{4 \pi} \sum_{\{i\}} \rho_{i^{\prime} i}^{F} \rho_{i i^{\prime}}^{V} .
$$


This implies that the matrix $\rho^{F}$ can be regarded as the density matrix for the production of $V$ in the rest frame of $F$. The matrix $\rho^{F}$ can be parametrized as follows [30]:

$$
\rho^{F}=\left(\begin{array}{ccc}
\frac{1}{3}+\frac{P_{z}}{2}+\frac{T_{z z}}{\sqrt{6}} & \frac{P_{x}-i P_{y}}{2 \sqrt{2}}+\frac{T_{x z}-i T_{y z}}{\sqrt{3}} & \frac{T_{x x}-T_{y y}-2 i T_{x y}}{\sqrt{6}} \\
\frac{P_{x}+i P_{y}}{2 \sqrt{2}}+\frac{T_{x z}+i T_{y z}}{\sqrt{3}} & \frac{1}{3}-\frac{2 T_{z z}}{\sqrt{6}} & \frac{P_{x}-i P_{y}}{2 \sqrt{2}}-\frac{T_{x z}-i T_{y z}}{\sqrt{3}} \\
\frac{T_{x x}-T_{y y}+2 i T_{x y}}{\sqrt{6}} & \frac{P_{x}+i P_{y}}{2 \sqrt{2}}-\frac{T_{x z}+i T_{y z}}{\sqrt{3}} & \frac{1}{3}-\frac{P_{z}}{2}+\frac{T_{z z}}{\sqrt{6}}
\end{array}\right) .
$$

In the above expression, $P_{i}$ and $T_{i j}(i=x, y, z)$ contain the information of the polarization of the vector boson $V . T_{i j}$ is a symmetric traceless tensor $\left(T_{x x}+T_{y y}+T_{z z}=0\right)$ and $P_{i}$ is a vector.

Substituting the expression for $\left(\frac{d \Gamma^{F f V}}{d \Omega^{F}}\right)_{F}$ and $\left(\frac{d \Gamma^{V} \ell \ell^{\prime}}{d \Omega_{\ell}^{V}}\right)_{V}$ in Eq. (28), we get

$$
\hat{\sigma}=\frac{1}{4 \pi} B \cdot R(F \rightarrow f V) B \cdot R\left(V \rightarrow \ell \bar{\ell}^{\prime}\right) \frac{f_{\text {avg }}^{V}}{4 \pi} \int \frac{d \hat{\sigma}_{F \bar{F}}}{d \bar{\Omega}} \operatorname{Tr}\left(R^{\dagger} \rho^{F} R \rho^{V}\right) d \Omega d \Omega_{\ell} d \bar{\Omega},
$$

where $d \Omega=\sin \theta d \theta d \phi, d \Omega_{\ell}=\sin \theta_{\ell} d \theta_{\ell} d \phi_{\ell}$ and $d \bar{\Omega}=\sin \bar{\theta} d \bar{\theta} d \bar{\phi}$. Evaluating the trace in Eq. (35), we get, after substituting the expressions for the matrices $\rho^{V}$ and $\rho^{F}$,

$$
\begin{aligned}
\frac{d \hat{\sigma}}{d \Omega_{\ell}}= & B . R .(F \rightarrow f V) B \cdot R\left(V \rightarrow \ell \bar{\ell}^{\prime}\right) \times \frac{f_{\mathrm{avg}}^{V}}{8 \pi} \iint\left(\frac{2}{3}-\frac{T_{z z}}{\sqrt{6}}\left(c_{\omega}^{2}-\frac{1}{2} s_{\omega}^{2}\right)\right)\left(\frac{d \hat{\sigma}_{F \bar{F}}}{d \bar{\Omega}}\right) \frac{d \Omega}{4 \pi} d \bar{\Omega} \\
& +\alpha c_{\theta_{\ell}} \int\left(\frac{d \hat{\sigma}_{F \bar{F}}}{d \bar{\Omega}}\right)\left(c_{\omega} P_{z}\right) \frac{d \Omega}{4 \pi} d \bar{\Omega}+c_{\theta_{\ell}}^{2} \sqrt{\frac{3}{2}} \int\left(\frac{d \hat{\sigma}_{F \bar{F}}}{d \bar{\Omega}}\right)\left(T_{z z}\left(c_{\omega}^{2}-\frac{1}{2} s_{\omega}^{2}\right)\right) \frac{d \Omega}{4 \pi} d \bar{\Omega} \\
& +\alpha s_{\theta_{\ell}} c_{\phi_{\ell}}\left(\int\left(\frac{d \hat{\sigma}_{F \bar{F}}}{d \bar{\Omega}}\right)\left(s_{\omega} c_{\chi} P_{z}\right) \frac{d \Omega}{4 \pi} d \bar{\Omega}\right)+\alpha s_{\theta_{\ell}} s_{\phi_{\ell}}\left(\int\left(\frac{d \hat{\sigma}_{F \bar{F}}}{d \bar{\Omega}}\right)\left(-s_{\omega} s_{\chi} P_{z}\right) \frac{d \Omega}{4 \pi} d \bar{\Omega}\right) \\
& +s_{\theta_{\ell}} c_{\theta_{\ell}} c_{\phi_{\ell}}\left(\int\left(\frac{d \hat{\sigma}_{F \bar{F}}}{d \bar{\Omega}}\right)\left(\sqrt{6} s_{\omega} c_{\omega} c_{\chi} T_{z z}\right) \frac{d \Omega}{4 \pi} d \bar{\Omega}\right)+s_{\theta_{\ell}} c_{\theta_{\ell}} s_{\phi_{\ell}}\left(\int\left(\frac{d \hat{\sigma}_{F \bar{F}}}{d \bar{\Omega}}\right)\left(-\sqrt{6} s_{\omega} c_{\omega} c_{\chi} T_{z z}\right) \frac{d \Omega}{4 \pi} d \bar{\Omega}\right) \\
& \left.+s_{\theta_{\ell}}^{2} s_{2 \phi_{\ell}}\left(\int\left(\frac{d \hat{\sigma}_{F \bar{F}}}{d \bar{\Omega}}\right)\left(-\frac{1}{4} \sqrt{\frac{3}{2}} s_{\omega}^{2} s_{2 \chi} T_{z z}\right) \frac{d \Omega}{4 \pi} d \bar{\Omega}\right)+s_{\theta_{\ell}}^{2} c_{2 \phi_{\ell}}\left(\int\left(\frac{d \hat{\sigma}_{F \bar{F}}}{d \bar{\Omega}}\right)\left(\frac{1}{4} \sqrt{\frac{3}{2}} s_{\omega}^{2} c_{2 \chi} T_{z z}\right) \frac{d \Omega}{4 \pi} d \bar{\Omega}\right)\right]
\end{aligned}
$$

where $c_{x}=\cos x, s_{x}=\sin x$. The angle $\omega$ is given by the following expressions:

$$
\cos \omega=\frac{(\beta+\bar{\beta} \cos \theta)}{\sqrt{\beta^{2}+\bar{\beta}^{2}-\beta^{2} \bar{\beta}^{2} \sin ^{2} \theta+2 \beta \bar{\beta} \cos \theta}}, \quad \sin \omega=\frac{\bar{\beta} \sin \theta}{\gamma \sqrt{\beta^{2}+\bar{\beta}^{2}-\beta^{2} \bar{\beta}^{2} \sin ^{2} \theta+2 \beta \bar{\beta} \cos \theta}} .
$$

Note that the angle $\omega$ is independent of the direction of motion of $F$ in the PCM frame, i.e., independent of $\bar{\theta}$ and $\bar{\phi}$. We have the following expressions for $\chi$ :

$$
\cos \chi=\cos \phi \cos \Delta \phi-\sin \Delta \phi \cos \bar{\theta} \sin \phi, \quad \sin \chi=-\frac{\sin \Delta \phi \sin \bar{\theta} \sqrt{\bar{\beta}^{2}+\beta^{2}-\bar{\beta}^{2} \beta^{2} \sin ^{2} \theta+2 \bar{\beta} \beta \cos \theta}}{\sqrt{1-\bar{\beta}^{2}} \beta \sin \theta},
$$

where $\Delta \phi=\bar{\phi}-\phi^{\mathrm{PCM}}, \phi^{\mathrm{PCM}}$ is a function of $\beta, \bar{\beta}, \theta, \phi$ and $\bar{\theta}$. The terms with $\cos \chi$ and/or $\sin \chi$ drop out after integration over the azimuthal angle $\phi_{\ell}$ of the lepton, since these terms always appear with factors such as $\sin \phi_{\ell}$, $\cos 2 \phi_{\ell}$, etc. Equation (36) becomes

$$
\begin{aligned}
\frac{d \hat{\sigma}}{d \cos \theta_{\ell}}= & 2 \pi \times B \cdot R \cdot(F \rightarrow f V) B \cdot R\left(V \rightarrow \ell \bar{\ell}^{\prime}\right) \times \frac{f_{\mathrm{avg}}^{V}}{8 \pi}\left[\int\left(\frac{2}{3}-\frac{T_{z z}}{\sqrt{6}}\left(c_{\omega}^{2}-\frac{1}{2} s_{\omega}^{2}\right)\right)\left(\frac{d \hat{\sigma}_{F \bar{F}}}{d \bar{\Omega}}\right) \frac{d \Omega}{4 \pi} d \bar{\Omega}\right. \\
& \left.+\alpha c_{\theta_{\ell}} \int\left(\frac{d \hat{\sigma}_{F \bar{F}}}{d \bar{\Omega}}\right)\left(c_{\omega} P_{z}\right) \frac{d \Omega}{4 \pi} d \bar{\Omega}+c_{\theta_{\ell}}^{2} \sqrt{\frac{3}{2}} \int\left(\frac{d \hat{\sigma}_{F \bar{F}}}{d \bar{\Omega}}\right)\left(T_{z z}\left(c_{\omega}^{2}-\frac{1}{2} s_{\omega}^{2}\right)\right) \frac{d \Omega}{4 \pi} d \bar{\Omega}\right] .
\end{aligned}
$$

The terms with $\cos \omega$ and $\sin \omega$ are independent of the direction of motion of $F(\bar{\theta}, \bar{\phi})$ in the PCM frame. This means that the integration $\int\left(d \hat{\sigma}_{F \bar{F}} / d \bar{\Omega}\right) d \bar{\Omega}$ can be performed independently to give a factor $\sigma_{F \bar{F}}$. The angle $\omega$ is also independent of the azimuthal angle $(\phi)$ of the vector boson $V$ in the rest frame of $F$. Hence, a partial integration of $d \Omega=d \cos \theta d \phi$ can be performed independently. The simplified Eq. (39) reads 


$$
\begin{aligned}
\frac{1}{\hat{\sigma}_{F \bar{F}}} \frac{d \hat{\sigma}}{d \cos \theta_{\ell}}= & B \cdot R \cdot(F \rightarrow f V) B \cdot R\left(V \rightarrow \ell \bar{\ell}^{\prime}\right) \frac{f_{\mathrm{avg}}^{V}}{4}\left[\int\left(\frac{2}{3}-\frac{T_{z z}}{\sqrt{6}}\left(c_{\omega}^{2}-\frac{1}{2} s_{\omega}^{2}\right)\right) \frac{1}{2} d \cos \theta+\alpha c_{\theta_{\ell}} \int\left(c_{\omega} P_{z}\right) \frac{1}{2} d \cos \theta\right. \\
& \left.+c_{\theta_{\ell}}^{2} \sqrt{\frac{3}{2}} \int\left(T_{z z}\left(c_{\omega}^{2}-\frac{1}{2} s_{\omega}^{2}\right)\right) \frac{1}{2} d \cos \theta\right] .
\end{aligned}
$$

Defining

$$
\begin{aligned}
\mathcal{T}_{z z}(\bar{\beta}) & =\left(\frac{1}{2} \int d \cos \theta\left(\cos ^{2} \omega-\frac{1}{2} \sin ^{2} \omega\right)\right) T_{z z}, \\
& =\frac{1}{4}\left(\int d \cos \theta\left(3 \cos ^{2} \omega-1\right)\right) T_{z z} \\
\mathcal{P}_{z}(\bar{\beta}) & =\left(\frac{1}{2} \int d \cos \theta \cos \omega\right) P_{z},
\end{aligned}
$$

we rewrite the above equation as

$$
\frac{1}{\hat{\sigma}_{F \bar{F}}} \frac{d \hat{\sigma}}{d \cos \theta_{\ell}}=B \cdot R \cdot(F \rightarrow f V) B \cdot R\left(V \rightarrow \ell \bar{\ell}^{\prime}\right) \frac{f_{\mathrm{avg}}^{V}}{4}\left[\frac{2}{3}-\frac{\mathcal{T}_{z z}(\bar{\beta})}{\sqrt{6}}+\alpha \cos \theta_{\ell} \mathcal{P}_{z}(\bar{\beta})+\sqrt{\frac{3}{2}} \cos ^{2} \theta_{\ell} \mathcal{T}_{z z}(\bar{\beta})\right]
$$

Comparing this expression with the expression for the azimuthal-averaged angular distribution of a decay product $\ell$ of a vector boson in its rest frame [24],

$$
\frac{1}{\sigma} \frac{d \sigma}{d \cos \theta_{\ell}}=\frac{f_{\mathrm{avg}}^{V}}{4}\left[\left(\frac{2}{3}-\frac{T_{z z}}{\sqrt{6}}\right)+\alpha P_{z} \cos \theta_{\ell}+\sqrt{\frac{3}{2}} T_{z z} \cos ^{2} \theta_{\ell}\right],
$$

where $\sigma$ is the cross section for the production of $V$, we interpret the quantities $\mathcal{P}_{z}(\bar{\beta})$ and $\mathcal{T}_{z z}(\bar{\beta})$ as the polarization parameters of $V$, for a given parton level event, as seen by a direct boost from the parton center of mass frame to the rest frame of $V$. Note that such a simplification would not have arisen had the factors involving $\chi$ been kept in Eq. (39), since they are functions of all the angular variables in the problem and hence the integration would have become a multidimensional one. In such a case, this method does not offer any advantage over a Monte Carlo simulation to extract the polarization parameters of $V$.

Before we complete the derivation of the expression of polarization estimators (see Sec. V), we discuss the expressions in Eq. (41). Performing the integrations over $\cos \theta$, we get

$$
\begin{aligned}
\mathcal{P}_{z}(\bar{\beta}) & =\frac{1}{2 \beta^{2} \bar{\beta}}\left[2 \bar{\beta}-\left(1-\beta^{2}\right) \log \left(\frac{1+\bar{\beta}}{1-\bar{\beta}}\right)\right] P_{z}(\bar{\beta}<\beta), \\
& =\frac{1}{2 \beta^{2} \bar{\beta}}\left[2 \beta-\left(1-\beta^{2}\right) \log \left(\frac{1+\beta}{1-\beta}\right)\right] P_{z}(\bar{\beta}>\beta), \\
& =\frac{1}{\beta^{3}}\left[\beta-\left(1-\beta^{2}\right) \frac{1}{2} \log \left(\frac{1+\beta}{1-\beta}\right)\right] P_{z}(\bar{\beta}=\beta),
\end{aligned}
$$

where $\bar{\beta}$ is the velocity of the parent particle in the PCM frame and $\beta$ is the velocity of the vector boson $V$ in the rest frame of the parent particle. Similarly, we get the expression for $\mathcal{T}_{z z}(\bar{\beta})$ :

$$
\begin{aligned}
& \mathcal{T}_{z z}(\bar{\beta})=\frac{T_{z z}}{8 \beta^{3} \bar{\beta}}\left[-4 \beta \bar{\beta}\left(-3+\beta^{2}\right)+3 \log \left(\frac{1-\beta \bar{\beta}}{1+\beta \bar{\beta}-\sqrt{1-\beta^{2}} \sqrt{1-\bar{\beta}^{2}} \sqrt{1-\bar{\beta}^{2}}}\right)\left(\left(2-\sqrt{\frac{1-\beta^{2}}{1-\bar{\beta}^{2}}}\right)\left(1-\beta^{2}\right)-\sqrt{1-\beta^{2}} \sqrt{1-\bar{\beta}^{2}}\right)\right. \\
& \left.-3 \log \left(\frac{1-\beta \bar{\beta}+\sqrt{1-\beta^{2}} \sqrt{1-\bar{\beta}^{2}}}{1+\beta \bar{\beta}+\sqrt{1-\beta^{2}} \sqrt{1-\bar{\beta}^{2}}}\right)\left(\left(2+\sqrt{\frac{1-\beta^{2}}{1-\bar{\beta}^{2}}}\right)\left(1-\beta^{2}\right)+\sqrt{1-\beta^{2}} \sqrt{1-\bar{\beta}^{2}}\right)\right], \quad(\bar{\beta} \neq \beta) \\
& =-\frac{T_{z z}}{2 \beta^{4}}\left[\beta^{2}\left(-3+\beta^{2}\right)-3\left(1-\beta^{2}\right) \log \left(1-\beta^{2}\right)\right], \quad(\bar{\beta}=\beta) \text {. }
\end{aligned}
$$




\section{A. Discussion}

We study the expressions Eqs. (44) and (45) for models of vectorlike quarks given in Sec. III where such decays are possible. We also consider the case of top decays in the SM. One can evaluate the value of $\mathcal{P}_{z}(\bar{\beta})$ and $\mathcal{T}_{z z}(\bar{\beta})$ at any given value of the velocity of the heavy fermion $\bar{\beta}$ using Eqs. (44) and (45) and the expression for $\beta$,

$$
\beta=\frac{K\left(1, \xi_{V}, \xi_{f}\right)^{1 / 2}}{1+\xi_{V}-\xi_{f}},
$$

obtained in the rest frame of $F$. Figure 1 shows the polarization parameters $P_{z}$ and $T_{z z}$ in the rest frame of $T(\bar{\beta}=0)$ as a function of $m_{T}$, for the two models given in Table I. One can see that, for large values of $m_{T}$, the value of $P_{z}$ tends to zero while that of $T_{z z}$ tends to a constant value. This can be understood from Eq. (4) (with $\xi_{V} \rightarrow \xi_{Z}$, $\left.\xi_{f} \rightarrow \xi_{t}\right)$. In the limit of large $m_{T},\left|P_{z}\right| \rightarrow 2 \xi_{Z} /\left(1-\xi_{t}\right) \rightarrow 0$ and $T_{z z} \rightarrow-\sqrt{2 / 3} \approx-0.82$, due to the fixed masses of $Z$ and $t$. Note that both $T_{z z}$ and $P_{z}$ are independent of the value of the mixing angles since one of the two couplings of $Z t T, g_{L}$ and $g_{R}$, is always zero for the two models considered in this work. This leads to results of $\mathcal{T}_{z z}(\bar{\beta})$ in the singlet model being identical to those of the doublet model, for any given value of $\bar{\beta}$ and $m_{T}$, which can be seen in the right panel of Fig. 1.

Figure 2 shows the values of polarization parameters $P_{z}(\bar{\beta})$ and $T_{z z}(\bar{\beta})$ for $\bar{\beta}=0.95$, for the two models. Comparing Fig. 2 with Fig. 1, one can see that the value of $T_{z z}$ significantly differs in the two cases, for each model, when the mass of the heavy fermion $T$ is low. Such a large difference is not observed in the case of $P_{z}$. In other words, the effect of the boost of the rest frame of $T$ relative to the PCM frame is more important in $T_{z z}$ than $P_{z}$. However, such effects decrease when the mass of $T$ becomes larger since the velocity $\beta$ of $Z$ in the rest frame of $T$ approaches unity (for a fixed $\bar{\beta}$ ). In the limit $\beta \rightarrow 1$, Eqs. (44) and (45) give $\mathcal{P}_{z}(\bar{\beta}) \rightarrow P_{z}$ and $\mathcal{T}_{z z}(\bar{\beta}) \rightarrow T_{z z}$, for a fixed $\bar{\beta}$. In other words, the values of $\mathcal{P}_{z}(\bar{\beta})$ and $\mathcal{T}_{z z}(\bar{\beta})$ tend to remain close to their values in the rest frame of $F$, for any value of $\bar{\beta}<\beta$, thus reducing their sensitivity to $\bar{\beta}(<\beta)$.

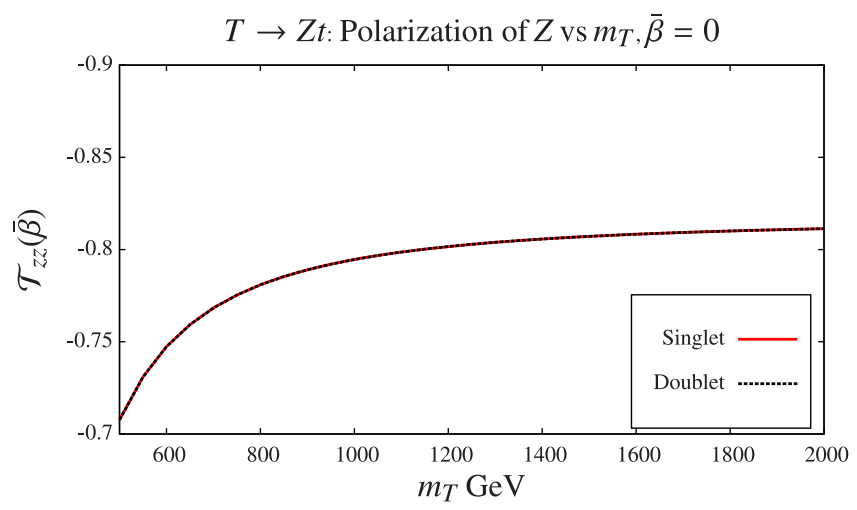

FIG. 1. The value of polarization parameters of $Z$, in the rest frame of $T$, as a function of $m_{T}$. The solid (dashed) lines correspond to the singlet (doublet) model. The remaining parameters of the two models are taken as in Table I. In the figure in the right panel, the lines corresponding to the two models are identical and appear merged.
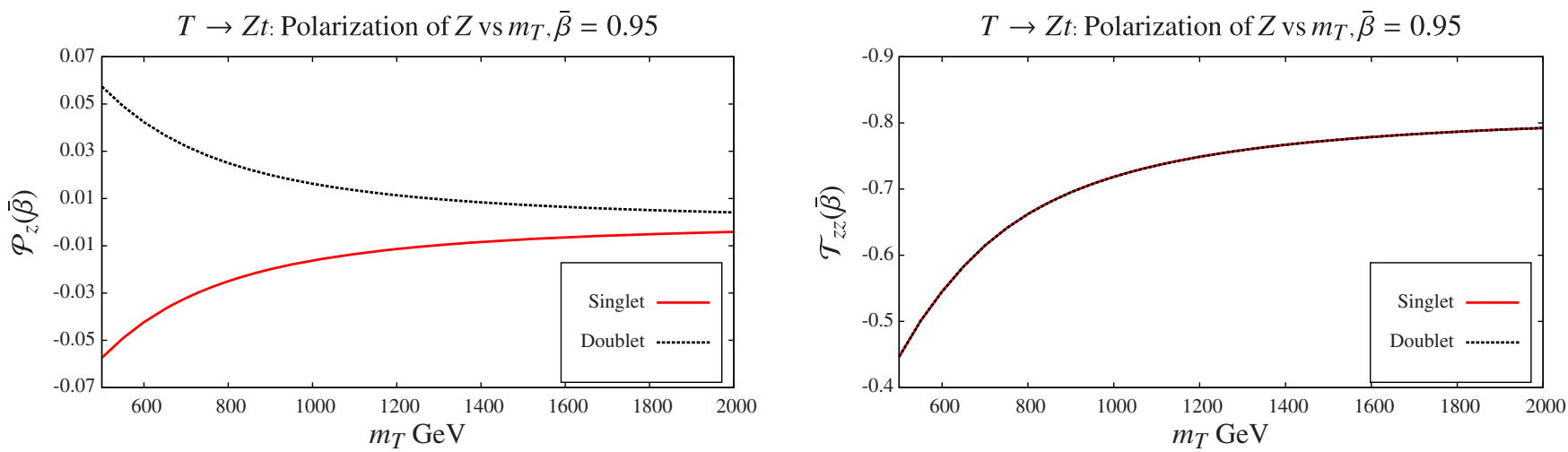

FIG. 2. The value of polarization parameters of $Z$ in a frame where $T$ moves with a velocity $\bar{\beta}=0.95$ as a function of $m_{T}$. The solid (dashed) lines correspond to the singlet (doublet) model. The remaining parameters of the two models are taken as in Table I. In the figure in the right panel, the lines corresponding to the two models are identical and appear merged. 

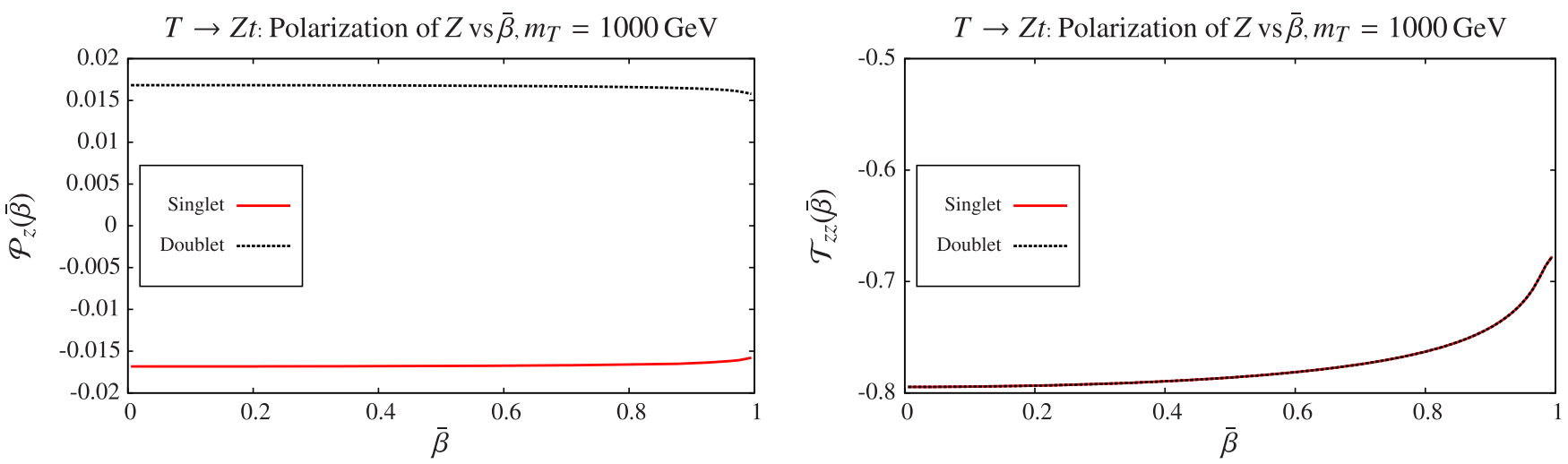

FIG. 3. The value of polarization parameters of $Z$ as a function of velocity $\bar{\beta}$ of the vectorlike quark $T$, with a mass $m_{T}=1000 \mathrm{GeV}$. The solid (dashed) lines correspond to the singlet (doublet) model. The remaining parameters of the two models are taken as in Table I. In the figure in the right panel, the lines corresponding to the two models are identical and appear merged.

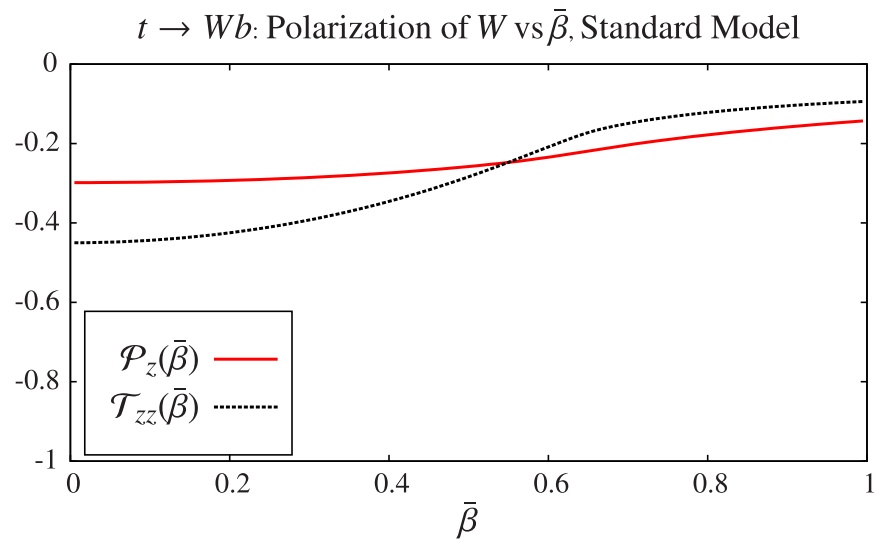

FIG. 4. The polarization parameters of $W$ in the decay of the top quark in the SM as a function of velocity of top quark $\bar{\beta}$. The solid (dashed) lines correspond to $\mathcal{P}_{z}(\bar{\beta})\left(\mathcal{T}_{z z}(\bar{\beta})\right)$.

The dependence of the polarization parameters $\mathcal{P}_{z}(\bar{\beta})$ and $\mathcal{T}_{z z}(\bar{\beta})$ is shown in Fig. 3, for the two models and for $m_{T}=1000 \mathrm{GeV}$. One can see that the effect of the boost of the rest frame of $T$ relative to the PCM frame $(\bar{\beta})$ is stronger at large values of $\bar{\beta}$, as it should be.

For completeness, in Fig. 4 we show the polarization parameters of $W$ in the top decay, in the SM, as a function of $\bar{\beta}$. In this case, due to the relatively lighter parent particle, the top, the dependence of $\mathcal{P}_{z}(\bar{\beta})$ and $\mathcal{T}_{z z}(\bar{\beta})$ on $\bar{\beta}$ (the velocity of the top in the PCM frame) are stronger even for moderate values of $\bar{\beta}$.

\section{POLARIZATION ESTIMATORS}

To obtain expressions for polarization parameters of $V$ at the level of $p p$ collisions, the expressions in Eqs. (44) and (45) on $\mathcal{P}_{z}(\bar{\beta})$ and $\mathcal{T}_{z z}(\bar{\beta})$ need to be convoluted over the parton distribution functions. This is required since the parton distribution functions and the cross section determine the $\bar{\beta}$ distribution in the PCM frame. Defining the cross section for the process $p p \rightarrow F \bar{F} \rightarrow \bar{F} f V \rightarrow \bar{F} f \ell \ell^{\prime}$ by

$$
\sigma=\int d x_{1} d x_{2} f_{p 1 / p}\left(x_{1}\right) f_{p 2 / p}\left(x_{2}\right) \hat{\sigma}
$$

where $\hat{\sigma}=\hat{\sigma}_{F \bar{F}} B \cdot R(F \rightarrow f V) B \cdot R\left(V \rightarrow \ell \ell^{\prime}\right)$, we get the expressions for the polarization parameters as

$$
\begin{aligned}
& \mathcal{P}_{z}^{\mathrm{NW}}=\frac{1}{\sigma} \int d x_{1} d x_{2} f_{p 1 / p}\left(x_{1}\right) f_{p 2 / p}\left(x_{2}\right) \mathcal{P}_{z}(\bar{\beta}), \\
& \mathcal{T}_{z z}^{\mathrm{NW}}=\frac{1}{\sigma} \int d x_{1} d x_{2} f_{p 1 / p}\left(x_{1}\right) f_{p 2 / p}\left(x_{2}\right) \mathcal{T}_{z z}(\bar{\beta}) .
\end{aligned}
$$

The superscript NW refers to the fact that we have used the NWA for $F$ (for $V$ it is applicable). This expression can also be written as an average over the $\bar{\beta}$ distribution as it is the only variable in the problem (the c.m. energy $\sqrt{\hat{s}}$ can be traded for $\bar{\beta}$ through $\bar{\beta}=\sqrt{1-4 m_{F}^{2} / \hat{s}}$ ).

$$
\begin{aligned}
& \mathcal{P}_{z}^{\mathrm{NW}}=\int \frac{1}{\sigma} \frac{d \sigma}{d \bar{\beta}} \mathcal{P}_{z}(\bar{\beta}) d \bar{\beta}, \\
& \mathcal{T}_{z z}^{\mathrm{NW}}=\int \frac{1}{\sigma} \frac{d \sigma}{d \bar{\beta}} \mathcal{T}_{z z}(\bar{\beta}) d \bar{\beta}
\end{aligned}
$$

where $1 / \sigma d \sigma / d \bar{\beta}$ is the normalized velocity distribution of $F$ in the PCM frame.

To convert the above expressions into the corresponding lab frame quantities, we replace the $\bar{\beta}$ distribution with the normalized velocity distribution of $F$ in the lab frame $1 / \sigma d \sigma / d \beta^{\text {lab }}$ and the polarization parameters by $P_{z}\left(\beta^{\text {lab }}\right)$ and $T_{z z}\left(\beta^{\text {lab }}\right)$ with $\beta^{\text {lab }}$ being the velocity of $F$ in the lab frame. In this case, the rest frame of $F$ is understood to be obtained by a direct boost of $\beta^{\text {lab }}$ from the lab frame. Hence, the final expressions for the polarization estimators of $V$ produced in the decay of $F$, in the lab frame are 

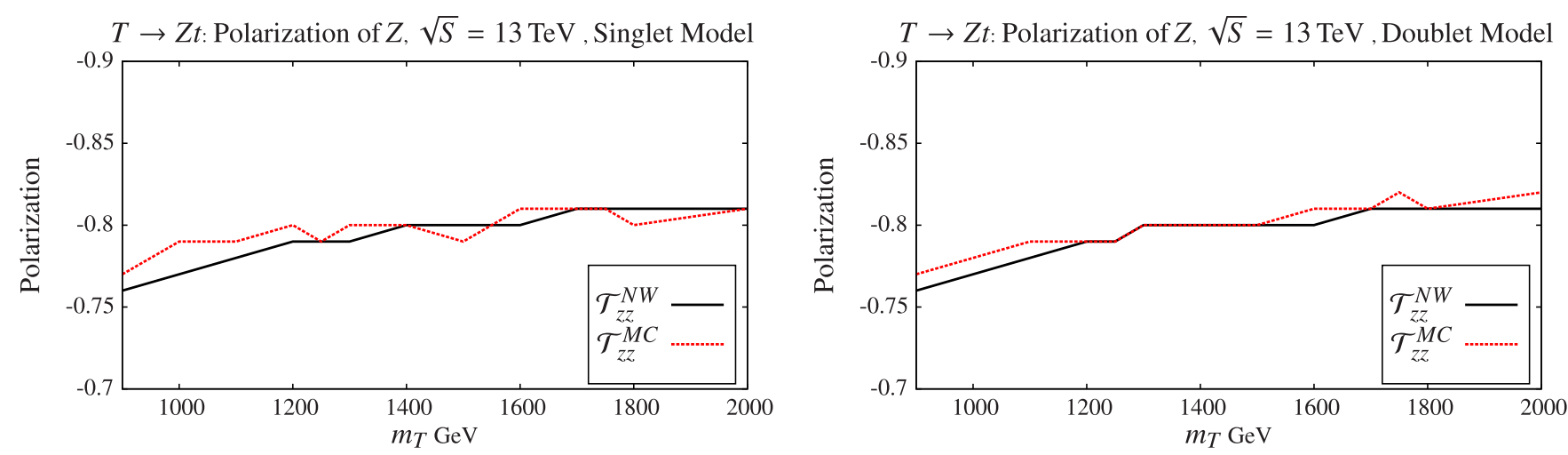

FIG. 5. A comparison of the two polarization estimators $\mathcal{T}_{z z}^{\mathrm{NW}}$ (solid) and $\mathcal{T}_{z z}^{\mathrm{MC}}$ (dashed) of $Z$ produced in the decay $T \rightarrow Z t$ for different choices of mass of $T\left(m_{T}\right)$. The left (right) panel corresponds to the singlet (doublet) model. The $p p$ collision center of mass energy $\sqrt{S}$ is taken as $13 \mathrm{TeV}$. The remaining parameters for the models are taken as in Table I.

$$
\begin{aligned}
& \mathcal{P}_{z}^{\mathrm{NW}}=\int \frac{1}{\sigma} \frac{d \sigma}{d \beta^{\mathrm{lab}}} \mathcal{P}_{z}\left(\beta^{\mathrm{lab}}\right) d \beta^{\mathrm{lab}}, \\
& \mathcal{T}_{z z}^{\mathrm{NW}}=\int \frac{1}{\sigma} \frac{d \sigma}{d \beta^{\mathrm{lab}}} \mathcal{T}_{z z}\left(\beta^{\mathrm{lab}}\right) d \beta^{\mathrm{lab}} .
\end{aligned}
$$

This equation can be interpreted as the average of $\mathcal{P}_{z}\left(\beta^{\text {lab }}\right)$ and $\mathcal{T}_{z z}\left(\beta^{\text {lab }}\right)$ over all the events with a weighting factor $(1 / \sigma) d \sigma / d \beta^{\text {lab }}$. We now present the numerical validation of the expressions for the polarization estimators of $V$ given in Eq. (50). We generate events for the process $p p \rightarrow T \bar{T}$ followed by the decay of $T$ into $Z$ and $t$ with $Z$ further decaying to $\ell \bar{\ell}$, using MadGraph [59]. The events correspond to the two models in Table I. For the models of $T$ concerned, due to the strong constraints on the couplings, the width of $T$ remains much smaller compared to its mass throughout the range of mass, i.e., 900 to $2000 \mathrm{GeV}$. This means that the NWA is a good approximation throughout the mass range considered and the expressions in Eq. (44) and Eq. (45) can be expected to be valid. We also considered the case of $W$ polarization in top decays both in the case of the singlet and doublet models and in the case of the SM. ${ }^{5}$ For this purpose, we generated events for $p p \rightarrow t \bar{t}$ and allowed the top to decay to $W$ and $b$ with $W$ further decaying to $\bar{\ell} \nu$. This provides an additional verification of our method. We use the value of velocity $\left(\beta^{\text {lab }}\right)$ of the heavy fermion ( $T$ or $t$ ) in the lab frame, from the generated sample and compute the quantities $\mathcal{P}_{z}\left(\beta^{\text {lab }}\right)$ and $\mathcal{T}_{z z}\left(\beta^{\text {lab }}\right)$ for each event and obtain an average over the entire event sample. This is equivalent to the use of Eq. (50). This method yields the

\footnotetext{
${ }^{5}$ The results for $W$ polarization in the case of the SM and in the case of the singlet model are not shown as they are identical to the other two cases. This is due to the fact that the $t b W$ couplings $\left(g_{L}\right.$ and $g_{R}$ ) in the two vectorlike quark models are very close to the corresponding SM values as a result of strong constraints on the $t-T$ and $b-B$ mixing angles.
}

values of $\mathcal{P}_{z}^{\mathrm{NW}}$ and $\mathcal{T}_{z z}^{\mathrm{NW}}$ since the heavy fermion is assumed to be on-shell due to the NWA.

The value of polarization parameters can be directly extracted from the Monte Carlo event samples through the use of angular asymmetries of the lepton $\ell$ from the $Z$ decay [24]. Consider the asymmetries $A_{z}$ and $A_{z z}$ defined by

$$
A_{z}=\frac{\sigma\left(\cos \theta_{\ell}>0\right)-\sigma\left(\cos \theta_{\ell}<0\right)}{\sigma\left(\cos \theta_{\ell}>0\right)+\sigma\left(\cos \theta_{\ell}<0\right)}
$$

and

$$
A_{z z}=\frac{\sigma\left(\sin 3 \theta_{\ell}>0\right)-\sigma\left(\sin 3 \theta_{\ell}<0\right)}{\sigma\left(\sin 3 \theta_{\ell}>0\right)+\sigma\left(\sin 3 \theta_{\ell}<0\right)},
$$

where $\theta_{\ell}$ is the polar angle of the lepton (from the $Z$ decay) in the rest frame of $Z$ that is obtained by a direct boost from the lab frame. $\sigma$ is the cross section for the production of $V$ followed by $V \rightarrow \ell \bar{\ell}^{\prime}$ and is the same as $\sigma$ in Eq. (47). These asymmetries can be directly related to the values of polarization parameters of $Z$ [see Eq. (43)]: performing the convolutions with parton distribution functions, we get

$$
\begin{aligned}
A_{z} & =\frac{3 \alpha}{4} \mathcal{P}_{z}^{\mathrm{MC}}, \\
T_{z z} & =\frac{3}{8} \sqrt{\frac{3}{2}} \mathcal{T}_{z z}^{\mathrm{MC}},
\end{aligned}
$$

where the MC superscript refers to the fact the polarization parameters are extracted from a Monte Carlo simulation. Note that in the case of $W$ produced in the top decay, the lepton $\ell$ corresponds to a neutrino ( $W \rightarrow \bar{\ell} \nu$ ), which is unobservable. Hence, one needs to use the asymmetries of $\bar{\ell}$ in place of the above-mentioned asymmetries of $\ell$. Since the neutrino and the anticharged lepton $\bar{\ell}$ have equal and opposite momenta, $\theta_{\bar{\ell}}=\pi-\theta_{\ell}$. This means that there is an additional factor of $(-1)$ in the first expression of Eq. (53). Figure 5 compares the value of polarization parameter $\mathcal{T}_{z z}^{\mathrm{NW}}$ described above and the direct extraction from Monte Carlo 

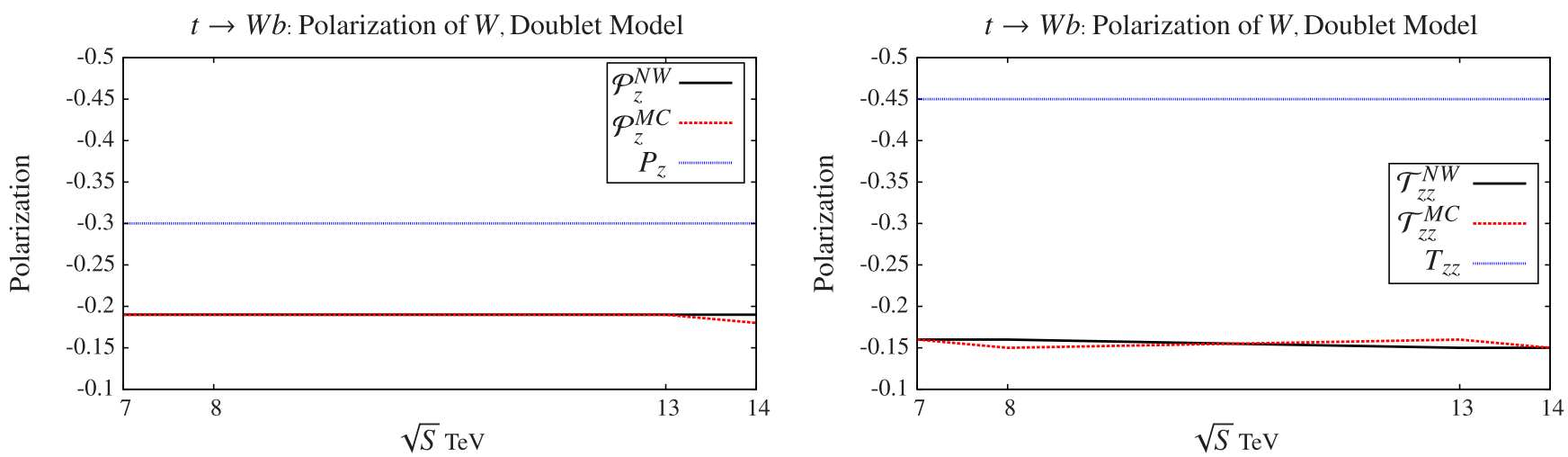

FIG. 6. A comparison of the polarization estimators of $W$ produced in the decay $t \rightarrow W b$ in the doublet model of vectorlike quark $T$, for different choices of $p p$ collision center of mass energy $\sqrt{S}$. The left (right) panel compares $\mathcal{P}_{z}^{\mathrm{NW}}$ (solid) and $\mathcal{P}_{z}^{\mathrm{MC}}$ (dashed) $\left[\mathcal{T}_{z z}^{\mathrm{NW}}\right.$ (solid) and $\mathcal{T}_{z z}^{\mathrm{MC}}$ (dashed)] along with the $W$ polarization in the top quark rest frame $P_{z}$ (dotted) $\left[T_{z z}\right.$ (dotted)]. The mass of $T$ ( $\left.m_{T}\right)$ is taken as $900 \mathrm{GeV}$. The remaining parameters for the models are taken as in Table I.

simulation $\mathcal{T}_{z z}^{\mathrm{MC}}$ for the decay $T \rightarrow Z t$. One can see that these values agree to within a few percent. The value of the other polarization parameters $\mathcal{P}_{z}^{\mathrm{NW}}, \mathcal{P}_{z}^{\mathrm{MC}}$ are not shown as their values are close to zero in both the models, as shown in Fig. 3. Their numerical values in both the models are provided in Table II. As an additional proof that our method is valid, we consider the decay of the top quark in the two models. The results are shown in Fig. 6, for the case of the doublet model, for four choices of $p p$ center of mass energy $\sqrt{S}$. One can see the excellent agreement between the two methods.

We have so far assumed that the width of the mother particle is small compared to its mass. This justified our application of the narrow width approximation by which we have taken the on-shell mass of the mother particle as its mass. Due to the strong constraints on the couplings of the vectorlike quarks, in the two models that are considered here the width of $T$ remains small $(\Gamma / m<0.02)$ compared to its mass throughout the mass range considered. This can be expected since the decays of $T$ are of electroweak type.

TABLE II. A comparison of polarization estimators $\mathcal{P}_{Z}^{\mathrm{NW}}$ and $\mathcal{P}_{z}^{\mathrm{MC}}$ of $Z$ produced in the decay $T \rightarrow Z t$, in the singlet and doublet models, for different values of the mass $\left(m_{T}\right)$ of the vectorlike quark $T$. The remaining parameters of the two models are taken as in Table I. The $p p$ center of mass energy $\sqrt{S}$ is taken as $13 \mathrm{TeV}$.

\begin{tabular}{|c|c|c|c|c|}
\hline \multirow[b]{2}{*}{$m_{T}(\mathrm{GeV})$} & \multicolumn{2}{|c|}{ Singlet } & \multicolumn{2}{|c|}{ Doublet } \\
\hline & $\mathcal{P}_{z}^{\mathrm{NW}}$ & $\mathcal{P}_{z}^{\mathrm{MC}}$ & $\mathcal{P}_{z}^{\mathrm{NW}}$ & $\mathcal{P}_{z}^{\mathrm{MC}}$ \\
\hline 900 & -0.02 & -0.02 & 0.02 & 0.01 \\
\hline 1000 & -0.02 & -0.02 & 0.02 & 0.02 \\
\hline 1250 & -0.01 & 0.01 & 0.01 & 0.02 \\
\hline 1500 & -0.01 & -0.01 & 0.01 & 0.00 \\
\hline 1750 & -0.01 & -0.01 & 0.01 & 0.01 \\
\hline 2000 & 0.00 & 0.00 & 0.00 & -0.01 \\
\hline
\end{tabular}

However, we indicate how to extend the applicability of our method to the case where the width of $T$ is large $(\Gamma / m \sim 0.1)$. In this case, nonresonant contributions to the process in question can not be neglected, in general. For example, the vector boson may come from a $t$-channel heavy fermion exchange rather than coming from the decay of the heavy fermion. The kinematics of such a process are different from the decay process we are interested in. Moreover, there are additional spin correlations between the production and the decay of the heavy fermion $T$ when the fermion is off-shell [60-62]. These off-shell effects are not present in the narrow width case, since the application of NWA results in on-shell vectorlike quark $F$. Hence, the extension of our method to the cases where $T$ has a finite width is, in general, highly nontrivial. However, in the case where the nonresonant production and the additional spincorrelation effects are small, we can construct appropriately modified polarization estimators. Since, in this case, the only additional effect is the smearing of mass of the parent particle, we take the invariant mass given by the fourmomentum carried by its propagator as its mass. This mass can then be used in expressions such as Eqs. (44) and (45), and the resulting polarization estimators are denoted as $\mathcal{P}_{z}^{\mathrm{BW}}$ and $\mathcal{T}_{z z}^{\mathrm{BW}}$, where the subscript BW refers to the BreitWigner shape of the propagator used in Monte Carlo simulations.

A comparison of the two sets of estimators is shown in Fig. 7, for the two models given in Table I with the widthto-mass ratio of $10 \%\left(\Gamma_{T} / m_{T}=0.1\right)$ for the vectorlike quark $T$. The corresponding plot for the other set of polarization estimators $\mathcal{P}_{z}^{\mathrm{NW}}, \mathcal{P}_{z}^{\mathrm{MC}}$ and $\mathcal{P}_{z}^{\mathrm{BW}}$ is not shown as their values are close to zero. One can see from Fig. 7 that both the estimators and $\mathcal{T}_{z z}^{\mathrm{BW}}$ agree with each other and with $\mathcal{T}_{z z}^{\mathrm{MC}}$ to within a few percent. This shows that the estimator derived in the narrow width case can also be used when the parent particle has a finite width. This is justified 

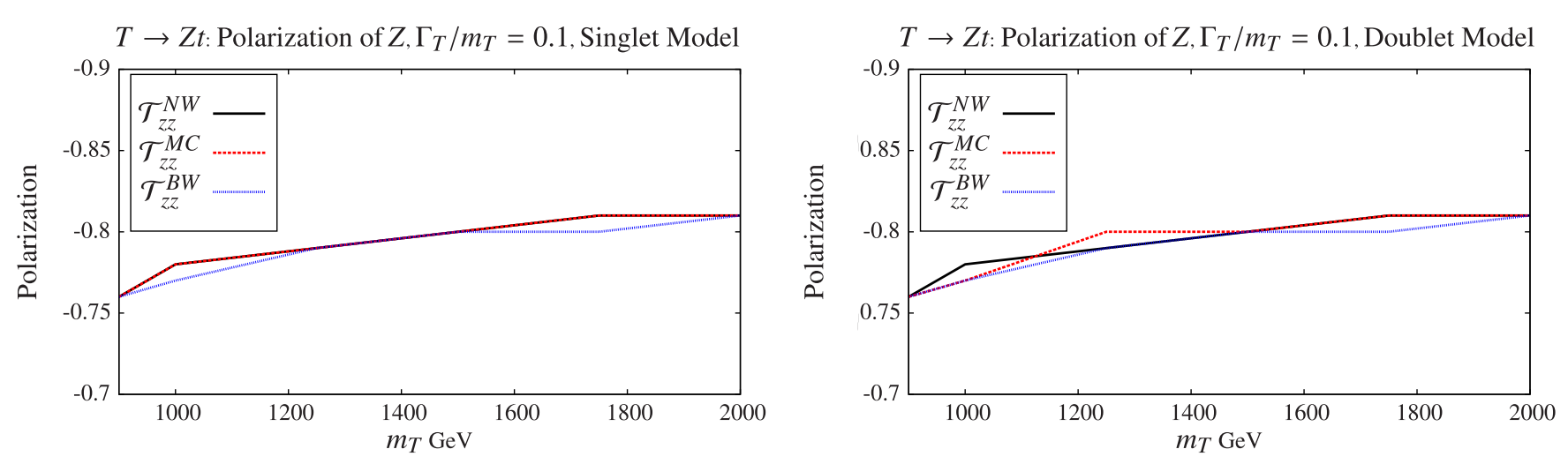

FIG. 7. A comparison of the polarization estimators $\mathcal{T}_{z z}^{\mathrm{NW}}$ (solid), $\mathcal{T}_{z z}^{\mathrm{MC}}$ (dashed) and $\mathcal{T}_{z z}^{\mathrm{BW}}$ (dotted) of $Z$ produced in the decay $T \rightarrow Z t$ for different choices of mass of $T\left(m_{T}\right)$. The left (right) panel corresponds to the singlet (doublet) model. The width-to-mass ratio $\Gamma_{T} / m_{T}$ of the vectorlike quark $T$ is taken to be 0.1 . The $p p$ collision center of mass energy $\sqrt{S}$ is taken as 13 TeV. The remaining parameters for the models are taken as in Table I.

provided we neglect any off-shell spin and nonresonant contributions.

\section{SUMMARY}

In this work, we obtained expressions for the polarization parameters of a vector boson $V$ (such as $W, Z$ ) produced in the decay of a heavy fermion such as a possible vectorlike quark or the top quark, both in the rest frame of the mother particle and in a frame where the mother particle is moving. Based on these expressions, we constructed simple estimators of the polarization parameters requiring only the velocity distribution of the mother particle, apart from the necessary couplings and masses involved in the decay of the mother particle. Since the vector boson has both a vector and a tensor polarization, we construct two nontrivial estimators, one for each type of polarization, which survive in the azimuthal-averaged decay distribution of the vector boson. The estimators $\mathcal{P}^{\mathrm{NW}}, \mathcal{T}_{z z}^{\mathrm{NW}}$ are derived under assumption that the width of the mother fermion is small compared its mass, applying the narrow width approximation for the mother particle. The advantage of this method is the possibility of a quick estimate of polarization parameters of the vector boson, in frames such as the lab frame. The polarization estimated by this method can be measured experimentally without any requirement to reconstruct any intermediate frame. We demonstrate the validity of this method in a set of models with a vectorlike quark and also in the case of the top decay in the SM.

We believe that this work will aid the study of the vectorlike quark phenomenology. This is because the polarization information carried by the vector boson from the decays of vectorlike quarks can be a probe of the coupling structure of the decay vertex. In addition to the case of vectorlike quarks of narrow width, we also consider the cases where the vectorlike quark has a finite width (width-to-mass ratio is taken to be 0.1). We restrict ourselves to the cases where the nonresonant production of the same final states and additional spin correlation between the production and decay of the vectorlike quark can be ignored. In this scenario, we propose two estimators, $\mathcal{P}_{z}^{\mathrm{BW}}$ and $\mathcal{T}_{z z}^{\mathrm{BW}}$, similar to the previous estimators by introducing an additional convolution over the BreitWigner shape of the vectorlike quark's invariant mass distribution. We validate the modified estimators and compare them with the original estimators. We find that both sets of estimators provide equally good approximations to the polarization parameters of $V$, in the finite width case.
[1] G. L. Kane, G. A. Ladinsky, and C. P. Yuan, Phys. Rev. D 45, 124 (1992).

[2] M. Jezabek and J.H. Kuhn, Phys. Lett. B 329, 317 (1994).

[3] R. M. Godbole, S. D. Rindani, and R. K. Singh, J. High Energy Phys. 12 (2006) 021.
[4] M. Baumgart and B. Tweedie, J. High Energy Phys. 08 (2013) 072.

[5] M. Perelstein and A. Weiler, J. High Energy Phys. 03 (2009) 141.

[6] M. Arai, K. Huitu, S. K. Rai, and K. Rao, J. High Energy Phys. 08 (2010) 082. 
[7] S. Gopalakrishna, T. Han, I. Lewis, Z.-g. Si, and Y.-F. Zhou, Phys. Rev. D 82, 115020 (2010).

[8] R. M. Godbole, K. Rao, S. D. Rindani, and R. K. Singh, J. High Energy Phys. 11 (2010) 144.

[9] B. Bhattacherjee, S. K. Mandal, and M. Nojiri, J. High Energy Phys. 03 (2013) 105.

[10] K.-i. Hikasa, J. M. Yang, and B.-L. Young, Phys. Rev. D 60, 114041 (1999).

[11] R. M. Godbole, L. Hartgring, I. Niessen, and C. D. White, J. High Energy Phys. 01 (2012) 011.

[12] D. Krohn, T. Liu, J. Shelton, and L.-T. Wang, Phys. Rev. D 84, 074034 (2011).

[13] S. D. Rindani and P. Sharma, J. High Energy Phys. 11 (2011) 082.

[14] D. Choudhury, R. M. Godbole, S. D. Rindani, and P. Saha, Phys. Rev. D 84, 014023 (2011).

[15] G. Belanger, R. M. Godbole, S. Kraml, and S. Kulkarni, arXiv:1304.2987.

[16] S. S. Biswal, S. D. Rindani, and P. Sharma, Phys. Rev. D 88, 074018 (2013).

[17] K. Huitu, S. Kumar Rai, K. Rao, S. D. Rindani, and P. Sharma, J. High Energy Phys. 04 (2011) 026.

[18] J. Cao, K. Hikasa, L. Wang, L. Wu, and J. M. Yang, Phys. Rev. D 85, 014025 (2012).

[19] S. Fajfer, J. F. Kamenik, and B. Melic, J. High Energy Phys. 08 (2012) 114.

[20] S. D. Rindani and P. Sharma, Phys. Lett. B 712, 413 (2012).

[21] M. M. Nojiri, Phys. Rev. D 51, 6281 (1995).

[22] J. A. Aguilar-Saavedra, J. Carvalho, N. F. Castro, F. Veloso, and A. Onofre, Eur. Phys. J. C 50, 519 (2007).

[23] J. A. Aguilar-Saavedra and J. Bernabeu, Nucl. Phys. B840, 349 (2010).

[24] R. Rahaman and R. K. Singh, Eur. Phys. J. C 76, 539 (2016).

[25] R. Rahaman and R. K. Singh, arXiv:1711.04551.

[26] G. Belanger, R. M. Godbole, L. Hartgring, and I. Niessen, J. High Energy Phys. 05 (2013) 167.

[27] J. Shelton, Phys. Rev. D 79, 014032 (2009).

[28] V. Arunprasath, R. M. Godbole, and R. K. Singh, Phys. Rev. D 95, 076012 (2017).

[29] C. Bourrely, J. Soffer, and E. Leader, Phys. Rep. 59, 95 (1980).

[30] F. Boudjema and R. K. Singh, J. High Energy Phys. 07 (2009) 028.

[31] A. Djouadi and A. Lenz, Phys. Lett. B 715, 310 (2012).

[32] G. Cacciapaglia, A. Deandrea, D. Harada, and Y. Okada, J. High Energy Phys. 11 (2010) 159.

[33] G. Cacciapaglia, A. Deandrea, L. Panizzi, N. Gaur, D. Harada, and Y. Okada, J. High Energy Phys. 03 (2012) 070.

[34] J. A. Aguilar-Saavedra, R. Benbrik, S. Heinemeyer, and M. Prez-Victoria, Phys. Rev. D 88, 094010 (2013).

[35] S. A. R. Ellis, R. M. Godbole, S. Gopalakrishna, and J. D. Wells, J. High Energy Phys. 09 (2014) 130.
[36] M. Carena, E. Ponton, J. Santiago, and C. E. M. Wagner, Nucl. Phys. B759, 202 (2006).

[37] K. Agashe, R. Contino, and A. Pomarol, Nucl. Phys. B719, 165 (2005).

[38] R. Contino, L. Da Rold, and A. Pomarol, Phys. Rev. D 75, 055014 (2007).

[39] R. Contino, T. Kramer, M. Son, and R. Sundrum, J. High Energy Phys. 05 (2007) 074.

[40] N. Arkani-Hamed, A. G. Cohen, and H. Georgi, Phys. Lett. B 513, 232 (2001).

[41] N. Arkani-Hamed, A. G. Cohen, T. Gregoire, and J. G. Wacker, J. High Energy Phys. 08 (2002) 020.

[42] N. Arkani-Hamed, A. G. Cohen, E. Katz, A. E. Nelson, T. Gregoire, and J. G. Wacker, J. High Energy Phys. 08 (2002) 021.

[43] N. Arkani-Hamed, A. G. Cohen, E. Katz, and A. E. Nelson, J. High Energy Phys. 07 (2002) 034.

[44] I. Low, W. Skiba, and D. Tucker-Smith, Phys. Rev. D 66, 072001 (2002).

[45] M. Perelstein, M. E. Peskin, and A. Pierce, Phys. Rev. D 69, 075002 (2004).

[46] ATLAS Collaboration, Report No. ATLAS-CONF-2015081, 2015.

[47] CMS Collaboration, Report No. CMS-PAS-EXO-15-004, 2015.

[48] S. Di Chiara, L. Marzola, and M. Raidal, Phys. Rev. D 93, 095018 (2016).

[49] R. Franceschini, G. F. Giudice, J.F. Kamenik, M. McCullough, A. Pomarol, R. Rattazzi, M. Redi, F. Riva, A. Strumia, and R. Torre, J. High Energy Phys. 03 (2016) 144.

[50] K. Harigaya and Y. Nomura, Phys. Lett. B 754, 151 (2016).

[51] D. Buttazzo, A. Greljo, and D. Marzocca, Eur. Phys. J. C 76, 116 (2016).

[52] J. Ellis, S. A. R. Ellis, J. Quevillon, V. Sanz, and T. You, J. High Energy Phys. 03 (2016) 176.

[53] S. Dawson and E. Furlan, Phys. Rev. D 86, 015021 (2012).

[54] A. M. Sirunyan et al. (CMS Collaboration), Phys. Lett. B 779, 82 (2018).

[55] A. M. Sirunyan et al. (CMS Collaboration), J. High Energy Phys. 11 (2017) 085.

[56] D. Berdine, N. Kauer, and D. Rainwater, Phys. Rev. Lett. 99, 111601 (2007).

[57] N. Kauer, Phys. Lett. B 649, 413 (2007).

[58] C. F. Uhlemann and N. Kauer, Nucl. Phys. B814, 195 (2009).

[59] J. Alwall, R. Frederix, S. Frixione, V. Hirschi, F. Maltoni, O. Mattelaer, H. S. Shao, T. Stelzer, P. Torrielli, and M. Zaro, J. High Energy Phys. 07 (2014) 079.

[60] R. Vega and J. Wudka, Phys. Rev. D 53, 5286 (1996); 56, 6037(E) (1997).

[61] A. Ballestrero and E. Maina, Phys. Lett. B 350, 225 (1995).

[62] P. Richardson, J. High Energy Phys. 11 (2001) 029. 